\title{
Magnetic field in IRC+10216 and other C-rich evolved stars
}

\author{
A. Duthu ${ }^{1}$, F. Herpin ${ }^{1}$, H. Wiesemeyer ${ }^{2}$, A. Baudry ${ }^{1}$, A. Lèbre ${ }^{3}$, and G. Paubert ${ }^{4}$ \\ ${ }^{1}$ Laboratoire d'Astrophysique de Bordeaux, Univ. Bordeaux, CNRS, B18N, Allée Geoffroy Saint-Hilaire, 33615 Pessac, France \\ e-mail: alizee.duthu@u-bordeaux.fr \\ 2 Max-Planck-Institut für Radioastronomie, Auf dem Hügel 69, 53121 Bonn, Germany \\ 3 Laboratoire Univers et Particules de Montpellier, CNRS et Université de Montpellier, Place E. Bataillon, 34090 Montpellier, France \\ ${ }^{4}$ Instituto Radioastronomía Milimétrica (IRAM), Av. Divina Pastora 7, Núcleo Central, 18012 Granada, Spain
}

Received 23 January 2017 / Accepted 10 May 2017

\begin{abstract}
Context. During the transition from the asymptotic giant branch (AGB) to planetary nebulae (PN), the circumstellar geometry and morphology change dramatically. Another characteristic of this transition is the high mass-loss rate, that can be partially explained by radiation pressure and a combination of various factors, such as the stellar pulsation, the dust grain condensation, and opacity in the upper atmosphere. The magnetic field can also be one of the main ingredients that shapes the stellar upper atmosphere and envelope. Aims. Our main goal is to investigate for the first time the spatial distribution of the magnetic field in the envelope of IRC +10216 . More generally we intend to determine the magnetic field strength in the circumstellar envelope (CSE) of C-rich evolved stars, compare this field with previous studies for O-rich stars, and constrain the variation of the magnetic field with $\mathrm{r}$ the distance to the star's centre.

Methods. We use spectropolarimetric observations of the Stokes $V$ parameter, collected with Xpol on the IRAM-30 m radiotelescope, observing the Zeeman effect in seven hyperfine components of the CN $J=1-0$ line. We use the Crutcher et al. (1996, ApJ, 456, 217) method to estimate the magnetic field. For the first time, the instrumental contamination is investigated, through dedicated studies of the power patterns in Stokes $V$ and $I$ in detail.

Results. For C-rich evolved stars, we derive a magnetic field strength $(B)$ between 1.6 and $14.2 \mathrm{mG}$ while $B$ is estimated to be $6 \mathrm{mG}$ for the proto-PN (PPN) AFGL618, and an upper value of $8 \mathrm{mG}$ is found for the PN NGC 7027. These results are consistent with a decrease of $B$ as $1 / r$ in the environment of AGB objects, that is, with the presence of a toroidal field. But this is not the case for PPN and PN stars. Our map of IRC+10216 suggests that the magnetic field is not homogeneously strong throughout or aligned with the envelope and that the morphology of the $\mathrm{CN}$ emission might have changed with time.
\end{abstract}

Key words. stars: evolution - polarization - stars: AGB and post-AGB - stars: carbon - stars: magnetic field - radio lines: stars

\section{Introduction}

Prior to the planetary nebula (PN) stage, stars ascend the asymptotic giant branch (AGB) phase and become thousands of times more luminous than on the main sequence. The central object experiences thermal pulses (Habing 1996). In addition, these stars lose significant amounts of their mass in the form of stellar winds. These outflows will form chemically rich circumstellar envelopes (CSEs) around the AGB stars. The more massive AGB stars exhibiting larger luminosities, longer pulsation periods, and enhanced mass losses also show strong $\mathrm{OH}$ maser emission and are referred to as $\mathrm{OH} / \mathrm{IR}$ stars. This transition from $\mathrm{AGB}$ to $\mathrm{PN}$ is hence characterised by a high mass-loss rate (e.g. De Beck et al. 2010). The mass-loss mechanism is driven mainly by the radiation pressure on the dust although a combination of several other factors may also play an important role (e.g. Höfner et al. 2016), including the stellar pulsation, the condensation and opacity of dust stellar grains in the upper atmosphere, and magnetic activity.

Moreover, during this stellar evolution, the star's geometry changes drastically; the quasi-spherical object inherited from the main sequence becomes axisymmetrical, point-like symmetrical, or even shows higher-order symmetries when reaching the proto-PN (PPN) and PN stage (e.g. Balick \& Franck 2002). The classical or generalized interacting stellar winds (GISW) models try to explain this shaping as the interaction between a slow AGB wind with a faster post-AGB wind. However, modelling of complex structures with peculiar jets is difficult. Moreover, this mechanism will amplify an initial asymmetry in the slow wind that has to be explained first. Understanding the launching of post-AGB jets is fundamental to understanding the post-AGB evolution. In several cases, the presence of a nearby companion might produce and maintain disks, jets, or an envelope rotation, and could explain how the shaping is launched (e.g. Akashi et al. 2015; Boffin et al. 2012). There are indications of stable, probably rotating disks in some post-AGB nebulae, mainly around binary stars (Alcolea et al. 2007; Bujarrabal \& Alcolea 2013).

Stellar magnetism can be one of the main ingredients in the shaping process. As a catalyst and/or as a collimating agent it could be the cause of a higher mass-loss rate in the equatorial plane, and thus could determine the global shaping of these objects (Blackman 2009). In addition, the ejection of massive winds by $A G B$ stars could be triggered by magnetic activity in the degenerated core as demonstrated with magneto-hydrodynamics (MHD) simulations (Pascoli \& Lahoche 2008, 2010).

Actually, there are several indications of the presence of a magnetic field at the stellar surface and in the CSE of these post-main sequence objects. In the Red Giant Branch (RGB) phase, magnetic fields have been detected. Aurière et al. (2015) obtained 29 Zeeman detections with Narval/ESPADOnS in a sample of active single G-K giants revealing, for the majority of them, a dynamo-type magnetic field. Konstantinova-Antova et al. (2014) have reported the detection of magnetic fields at and 
Table 1. Source list and observation parameters.

\begin{tabular}{lccccccccc}
\hline \hline Object & Type & $\begin{array}{c}\text { RA } \\
{[\mathrm{h} \mathrm{m} \mathrm{s}]}\end{array}$ & $\begin{array}{c}\text { Dec }^{a} \\
{\left[{ }^{\prime}{ }^{\prime \prime}\right]}\end{array}$ & $\begin{array}{c}d \\
{[\mathrm{pc}]}\end{array}$ & $\begin{array}{c}V_{\mathrm{LSR}} \\
{\left[\mathrm{km} \mathrm{s}^{-1}\right]}\end{array}$ & $\begin{array}{c}L_{\mathrm{bol}} \\
{\left[10^{3} L_{\odot}\right]}\end{array}$ & $\begin{array}{c}\dot{M} \\
{\left[10^{-6} M_{\odot} / \mathrm{yr}\right]}\end{array}$ & $\begin{array}{c}T_{\text {sys }} \\
{[\mathrm{K}]}\end{array}$ & $\begin{array}{c}\mathrm{rms} \\
{[\mathrm{mK}]}\end{array}$ \\
\hline IRC+10216 & AGB & 094757.38 & +131643.7 & $120^{d}$ & -25.5 & $9.8^{d}$ & $10-40^{e}$ & $200-220$ & $12-14$ \\
RW LMi & AGB & 101602.35 & +303419.0 & $400^{d}$ & -1.6 & $10^{d}$ & $5.9^{f}$ & $200-260$ & 12 \\
RY Dra & AGB & 125625.91 & +655939.8 & $431^{d}$ & -7.3 & $4.5^{d}$ & $0.2^{g}$ & $290-360$ & 60 \\
AFGL618 & PPN & 044253.67 & +360653.2 & $900^{a}$ & -25.0 & $10-14^{b}$ & $20-110^{c}$ & 200 & 14 \\
NGC 7027 & PN & 210701.59 & +421410.2 & $980^{h}$ & 25.0 & $7.2^{h}$ & N/A & $230-250$ & 14 \\
\hline
\end{tabular}

Notes. The rms is for a velocity resolution of $0.2 \mathrm{~km} \mathrm{~s}^{-1}$ in Stokes $I .{ }^{(a)}$ Sanchez Contreras \& Sahai (2004). ${ }^{(b)}$ Knapp et al. (1993). ${ }^{(c)}$ Lee et al. (2013a,b). ${ }^{(d)}$ Ramstedt \& Olofsson (2014). ${ }^{(e)}$ De Beck et al. (2012). ${ }^{(f)}$ De Beck et al. (2010). ${ }^{(g)}$ Cox et al. (2012). ${ }^{(h)}$ Zijlstra et al. (2008).

above the Gauss level in approximately $50 \%$ of their RGB/AGB sample demonstrating that the magnetic field is commonly detected at the surface of these objects. Moreover, several studies have revealed the presence of a strong magnetic field in the CSE of AGB, post-AGB stars, and PNe. For O-rich AGB objects, the magnetic field strength is estimated from the polarised maser emission of several molecules, located at different distances from the central star: $B \sim 0-18 \mathrm{G}$ with mean value of $3.5 \mathrm{G}$ in the inner part at 5-10 AU (SiO masers, Herpin et al. 2006), a few $100 \mathrm{mG}$ at $100 \mathrm{AU}$ (water masers, Vlemmings et al. 2001; Leal-Ferreira et al. 2013), and around $10 \mathrm{mG}$ in the outer part at 1000-10000 AU (OH masers, Kemball \& Diamond 1997; Rudnitski et al. 2010). Recently, for the first time the magnetic field strength has been estimated to be about $2-3 \mathrm{G}$ at the surface of the S-type Mira star $\chi$ Cyg (Lèbre et al. 2014). Moreover, direct observational evidence of large-scale magnetic fields at the surface and in the environment of Post-AGB stars has recently been established (Sabin et al. 2007, 2014, 2015). The post-AGB study (from OH masers) of Gonidakis et al. (2014) indicates that the $B$ strength detected in CSEs increases with the post-AGB age. While no strong magnetic field has been detected in the central stars of PNe (Jordan et al. 2012; Leone et al. 2014; Steffen et al. 2014), large-scale fields have been observed in their nebulae (Gómez et al. 2009) and are shown to be responsible for the observed small-scale structures.

Indications for a link between morphological structures and the magnetic field exist. Lèbre et al. (2014) have underlined a connection between the surface magnetic field and the atmospheric shock waves in $\chi$ Cyg, a star exhibiting a departure from spherical symmetry at the photospheric level (Ragland et al. 2006). The MHD-dust-driven modelling of Thirumalai \& Heyl (2012) shows that the magnetic field certainly plays a role in the mass-loss process in the equatorial plane of the Mira star o Ceti. In the $\mathrm{OH} / \mathrm{IR}$ star $\mathrm{OH} 231.8+4.2$, a binary system, the magnetic vectors follow the molecular outflows (Sabin et al. 2014), the field strength being estimated to be $2.5 \mathrm{G}$ at the stellar surface (Leal-Ferreira et al. 2012). The same authors have shown a clear correlation between field orientation (toroidal field oriented along the equatorial torus) and the nebular structure of the PN NGC 7027 (Sabin et al. 2007).

As for AGB stars, the origin of the field detected in the CSEs remains unclear, as well as its possible variability with time. All of the measurements performed so far throughout AGB CSEs (see Vlemmings 2012) favour a $1 / r$ law for the radial dependence of a toroidal magnetic field. Pascoli (1997) and Pascoli \& Lahoche $(2008,2010)$ proposed that a toroidal magnetic field of $\sim 10^{6} \mathrm{G}$ is produced by a dynamo mechanism in the degenerate core and results in a field strength of a few tens of Gauss on the stellar surface. On the other hand, the polarisation morphology of $\mathrm{SiO}$ masers in the CSE of an AGB star has been investigated by Assaf et al. (2013) using the Very Long Baseline Array and appears to be consistent with a radial magnetic field.

Another condition for the field to be able to shape stars is that it must be sustained over the AGB lifetime. This implies either the presence of a companion to spin up the envelope of the star, thus providing the missing angular momentum (Nordhaus et al. 2007; Blackman 2009), or that the differential rotation between the core and the envelope of the star has to be re-supplied via convection or another mechanism.

In addition, some uncertainties remain concerning the magnetic field obtained throughout the CSEs of evolved stars. Indeed, the magnetic field strength (B) derived from $\mathrm{SiO}$ masers is still debatable because anisotropic pumping can produce strong polarised maser emission (Western \& Watson 1983; Desmurs et al. 2000) and magnetic field strengths of approximately $15 \mathrm{mG}$ which might be sufficient to explain the observational results (Houde 2014). Hence, using field tracers other than $\mathrm{SiO}$ is crucial in order to have a reliable estimate of $\mathrm{B}$ and of its variation throughout the envelope. Moreover, most previous studies have focused on O-rich stars and AGB objects and similar studies should be conducted for C-rich objects. However, the main probe close to the stellar atmosphere, $\mathrm{SiO}$ maser emission, is only present in O-rich evolved objects and disappears soon after the star has reached the end of the AGB phase (Nyman et al. 1998). As a consequence, other magnetic field probes, such as the $\mathrm{CN}$ radical used in this work, are most useful to study more advanced stages of the stellar evolution or C-rich objects.

In this paper we present $\mathrm{CN}$ Zeeman observations of several C-rich objects at different evolutionary stages. We first study the distribution of the magnetic field in IRC+10216 and then present the result for four other C-rich evolved stars to compare. Sections 2 and 3 present our source sample and observations, respectively. Details on CN Zeeman splitting and on data analysis are given in Sect. 4. Results from the Zeeman interpretation are given in Sect. 5. We finally discuss the importance of the magnetic field and compare our results with previous studies in Sect. 6.

\section{Source sample}

Our source sample consists of five evolved low- or intermediatemass carbon stars: three AGB objects (IRC+10216, RY Dra, and RW LMi), one proto-PN (AFGL618), and one PN (NGC 7027). The stellar parameters (coordinates, distance, LSR velocity, bolometric luminosity, and estimated mass-loss rate) are given in Table 1 and each source is presented below. Most of these sources have already been observed in $\mathrm{CN}$ by several authors; 
Table 2. For $\mathrm{CN}$ layer, molecular abundance relative to $\mathrm{H}_{2}$, its distance $d_{\mathrm{CN}}$ to the central star, and stellar radius for each object in our sample.

\begin{tabular}{lccc}
\hline \hline Object & {$\left[\mathrm{CN} / \mathrm{H}_{2}\right]$} & $\begin{array}{c}d_{\mathrm{CN}} \\
{[\mathrm{AU}]}\end{array}$ & $\begin{array}{c}R_{*} \\
{[\mathrm{AU}]}\end{array}$ \\
\hline RW LMi & $3 \times 10^{-5}$ & $2675-3340\left(3-9^{\prime \prime}\right)$ & 2.6 \\
RY Dra & $5.1 \times 10^{-5}$ & $61-615(0.14-1.5)^{\prime \prime}$ & 1.0 \\
IRC+10216 & $8.0 \times 10^{-6}$ & $2500\left(21^{\prime \prime}\right)$ & 3.3 \\
AFGL618 & $2.1 \times 10^{-6}$ & $2700\left(3^{\prime \prime}\right)$ & 0.24 \\
NGC 7027 & $2.3 \times 10^{-7}$ & $10000\left(11^{\prime \prime}\right)$ & $3.5 \times 10^{-4}$ \\
\hline
\end{tabular}

Notes. From the literature, see Sect. 2.

we try to estimate the size of the $\mathrm{CN}$ layer and the distance to the central object for each star (see Table 2).

IRC +10216 is one of the closest AGB stars (120 pc, period of 630 days, Ramstedt \& Olofsson 2014) and the beststudied C-rich object. Half of the known interstellar species are observed in its outer envelope. It is surrounded by an optically thick C-rich CSE, which results from the ejection of stellar material at a rate of $1-4 \times 10^{-5} M_{\odot} / \mathrm{yr}$ (De Beck et al. 2012). At first glance, IRC+10216 is nearly spherical and expands radially (over more than $450^{\prime \prime}$ in $\mathrm{CO}$ ) with a velocity of $14.5 \mathrm{~km} \mathrm{~s}^{-1}$ (Cernicharo et al. 2015). Deviations from symmetry are visible at small angular scales (e.g. Skinner et al. 1998), suggesting the presence of an overall bipolar structure. Moreover, high-angular-resolution observations indicate the presence of clumps and show that the innermost structures at subarsecond scale are changing on timescales of years (e.g. Tuthill et al. 2000), and that this object might have begun its evolution towards the PN phase. ALMA observations by Decin et al. (2015) have revealed that the bipolar structure with concentric shells is indeed a binary-induced spiral shell. A faint companion of the AGB star might have been discovered by Kim et al. (2015) and may explain the observed circumstellar geometry. However, a cyclic magnetic activity at the stellar surface, similar to that on the Sun, is an alternative explanation (Soker 2000). Investigating the magnetic field strength in IRC+10216 might help to discriminate between these two alternatives. A large $40^{\prime \prime}$ diameter ( 5000 AU) $\mathrm{CN}$ ring with two symmetrical brighter emission lobes has been mapped with the IRAM interferometer (Lucas et al. 1995). In addition, Lindqvist et al. (2000) estimated the $\mathrm{CN}$ abundance at $8 \times 10^{-6}$ and inferred a $\mathrm{CN}$ ring with a radius of $5 \times 10^{16} \mathrm{~cm}(\sim 3300 \mathrm{AU})$ and a width of $4 \times 10^{16} \mathrm{~cm}$.

Two other C-rich AGB objects, RW LMi and RY Dra, are studied in addition to IRC+10216. RW LMi, also known as CIT6, is an M-type C-rich star (period of 640 days, Ramstedt \& Olofsson 2014) with a very rich CSE (Schmidt et al. 2002), believed to be in transition from the AGB to the post-AGB phase. Schmidt et al. (2002) found the presence of a nascent bipolar nebula, providing evidence that the evolutionary phase of CIT6 lies just past the tip of AGB. Like IRC+10216, a spiral structure has been discovered in the circumstellar envelope. It is likely induced by a central binary star (Kim et al. 2013), and extends over 20". In fact, the molecular content of the RW LMi envelope suggests that this object is more evolved than IRC+10216 (Chau et al. 2012). The interferometric observations of Lindqvist et al. (2000) reveal a $\mathrm{CN}$ ring with a radius of 2675-3340 AU. RY Dra belongs to the J-type carbon stars (i.e. stars with ${ }^{12} \mathrm{C} /{ }^{13} \mathrm{C}$-ratios $\sim 3$ ). This object is a b-type semi-variable (period $=173$ days, Ramstedt \& Olofsson 2014). A detached shell has been revealed by the ISO observations of Izumiura \& Hashimoto (1999) and could have been produced by a previous episode of mass loss. Neither interferometric data nor Hubble images are available for this object. The PACS Herschel map (Herschel archive) of this object does not spatially resolve the spherical circumstellar envelope whose size appears to be a few arcsec. Only CN single-dish observations are available for RY Dra (Bachiller et al. 1997a), which lead to a CN abundance of $5.1 \times 10^{-5}$ and an estimated $\mathrm{CN}$ ring size of $0.14-1.5^{\prime \prime}(61-$ 615 AU).

AFGL618, also named the Westbrook Nebula, is a young proto-PN ( 200 years Kwok \& Bignell 1984) exhibiting a B0 central star, a central compact HII region (Sánchez Contreras et al. 2002), and two pairs of rapidly expanding well collimated lobes (Balick et al. 2013). SMA continuum and CO-line-polarisation observations of Sabin et al. (2014) have revealed a magnetic field well aligned and organised along the polar direction, suggesting a magnetic-outflowlaunching mechanism. The extended ( $\left.5^{\prime \prime}\right)$ molecular envelope is composed of material ejected during the AGB phase and partly chemically reprocessed (e.g. Herpin et al. 2002). The molecular outflows extend over 20" (Sánchez Contreras et al. 2002). Two episodes of collimated fast winds have been identified (Lee et al. 2013): one with a huge mass-loss rate of $\sim 1.1 \times 10^{-4} M_{\odot} \mathrm{yr}^{-1}$, and an older one with $\sim 2 \times 10^{-5} M_{\odot} \mathrm{yr}^{-1}$. The $\mathrm{CN}$ abundance $\left(2.1 \times 10^{-6}\right)$ has been derived by Bachiller et al. (1997b). To estimate the $\mathrm{CN}$ distribution and abundance in this object, only a HCN map is available (Sánchez Contreras et al. 2002). Since $\mathrm{HCN}$ is a molecule of photospheric origin that becomes photodissociated by the ambient interstellar UV-field into CN (e.g. Huggins \& Glassgold 1982), we can assume the CN molecules are surrounding the $\mathrm{HCN}$ envelope, leading to a rough estimate of the CN envelope size of $6^{\prime \prime}$ in diameter, hence $8.1 \times 10^{16} \mathrm{~cm}$ (or $5400 \mathrm{AU}$ ).

NGC 7027 is a young PN (kinematical age of 600 years, Masson 1989), with a high degree of axial symmetry. Recent studies have shown that this object is a multipolar PN in the making (see Huang et al. 2010). High-velocity jets exhibit a multipolar shape in $\mathrm{H}_{2}$ extending over roughly $20^{\prime \prime}$ (Sabin et al. 2007). A spherical CO envelope extends over $60^{\prime \prime}$ around the central HII region. While the inner part of the torus has been ionised, the ancient AGB molecular content has been completely reprocessed in the envelope (Herpin et al. 2002). The HCN emission extends over 20" (Huang et al. 2010). We had access to an estimate of the CN distribution from Plateau de Bure observations (Josselin et al., priv. commun.,) showing $\mathrm{CN}$ at a distance of $10000 \mathrm{AU}$ from the central object.

\section{Observations}

We have obtained simultaneous spectroscopic measurements of the four Stokes parameters $I, U, Q, V$ for the seven $C N 1-0$ hyperfine transitions given in Table 3 . Two observing runs have been performed, the first one in November 2006 and the second one in March and June 2016. The observations were carried out with the XPol polarimeter (Thum et al. 2008) at the IRAM-30 m telescope on Pico Veleta, Spain.

For the first observing session (November 2006), the pointing was regularly checked directly on the observed stars themselves. The system temperature of the SIS receiver and the rms are given in Table 1 . The front-ends were the facility receivers A100 and B100, and the back-end was the versatile spectrometer array (VESPA) correlator. The lines were observed with a spectral resolution of $0.11 \mathrm{~km} \mathrm{~s}^{-1}(40 \mathrm{kHz})$ in order to observe the Zeeman effect with sufficient accuracy. All hyperfine component lines were simultaneously covered with two VESPA sections of 
Table 3. Zeeman splitting factor $Z$ for $\mathrm{CN} \mathrm{N}=1 \rightarrow 0$ hyperfine components (Crutcher et al. 1996).

\begin{tabular}{cccccc}
\hline \hline$\#$ & $N_{J^{\prime}, F^{\prime}}^{\prime} \rightarrow N_{J, F}$ & $\begin{array}{c}v_{0} \\
{[\mathrm{GHz}]}\end{array}$ & $\begin{array}{c}Z \\
{[\mathrm{~Hz} / \mu \mathrm{G}]}\end{array}$ & $R I$ & $Z \times R I$ \\
\hline 1 & $1_{1 / 2,1 / 2} \rightarrow 0_{1 / 2,3 / 2}$ & 113.14434 & 2.18 & 8 & 17.4 \\
2 & $1_{1 / 2,3 / 2} \rightarrow 0_{1 / 2,1 / 2}$ & 113.17087 & -0.31 & 8 & -2.5 \\
3 & $1_{1 / 2,3 / 2} \rightarrow 0_{1 / 2,3 / 2}$ & 113.19133 & 0.62 & 10 & 6.2 \\
\hline 4 & $1_{3 / 2,3 / 2} \rightarrow 0_{1 / 2,1 / 2}$ & 113.48839 & 2.18 & 10 & 21.8 \\
5 & $1_{3 / 2,5 / 2} \rightarrow 0_{1 / 2,3 / 2}$ & 113.49115 & 0.56 & 27 & 15.1 \\
6 & $1_{3 / 2,1 / 2} \rightarrow 0_{1 / 2,1 / 2}$ & 113.49972 & 0.62 & 8 & 5.0 \\
7 & $1_{3 / 2,3 / 2} \rightarrow 0_{1 / 2,3 / 2}$ & 113.50906 & 1.62 & 8 & 13.0 \\
\hline
\end{tabular}

Notes. $R I$ stands for Relative Intensity in local thermodynamic equilibrium (LTE) conditions. Two CN groups are distinguished: lines 1-3 and 4-7.

80 and $40 \mathrm{MHz}$ width, respectively, each one being correctly centred. The integration times (pointed, wobbler-switched observations) were 137, 175, 248, 17, and $215 \mathrm{~min}$, for AFGL618, IRC+10216, RW LMi, RY Dra, and NGC 7027, respectively. The forward and main beam efficiencies were 0.95 and 0.74 , respectively, at the $\mathrm{CN}$ frequencies, while the half-power beam width was $21.7^{\prime \prime}$. The Jy/K conversion factor is 6.3 . The polarisation angle calibration (see Thum et al. 2008) has been verified by means of observations of the Crab Nebula. Moreover, planets have been used to check the instrumental polarisation along the optical axis (their intrinsic polarisation is negligible at the considered frequency, or, for Mars and Mercury, cancels out in the beam). A more detailed discussion about any instrumental effect is given in Sect. 5.1.

The 2016 observations were exclusively dedicated to IRC +10216 . The main goal was to map the magnetic field in the $\mathrm{CN}$ envelope that is resolved by the beam of the telescope. In addition to the positions observed in $2006\left(\left(0^{\prime \prime}, 0^{\prime \prime}\right)\right.$, corresponding to the central source position, and $\left.\left(-10^{\prime \prime},-20^{\prime \prime}\right),\left(+15^{\prime \prime},-15^{\prime \prime}\right)\right)$ we observed four offset positions $\left(-18^{\prime \prime},-10^{\prime \prime}\right),\left(-18^{\prime \prime},+10^{\prime \prime}\right)$, $\left(+18^{\prime \prime},-04^{\prime \prime}\right)$, and $\left(+20^{\prime \prime},+16^{\prime \prime}\right)$. The EMIR band E090, with the backend VESPA, was set up to observe all CN 1-0 hyperfine components simultaneously, as with the facility receiver in 2006, but now with larger bandwidths ( 160 and $80 \mathrm{MHz}$ ) in order to better define the spectral baseline. The focus and the pointing were checked on Jupiter. A major change occurred at the telescope during the winter before our 2016 observations. The EMIR $3 \mathrm{~mm}$ band was upgrated in November 2015. The mixer was exchanged, but also the dual-horn system was changed to a single horn system. The linear horizontal and vertical polarisation splitting are not obtained from a grid anymore but from an orthomode transducer. The receiver has therefore one single horn followed by a wave guide wherein the signal is separated into horizontal and vertical components. In Sect. 4.2.3 we discuss the impact of this new design on our 2016 observations, that is, a leakage of the Stokes $I$ into the $V$ signal. One of the consequences is that we had to increase the integration time per point (on average $11 \mathrm{~h}$ by position compared to $1 \mathrm{~h}$ before) to retrieve a sufficient $S / N$ in the $V$ signal.

\section{Data analysis and method}

\section{1. $C N$ and magnetic field}

Observing $\mathrm{CN}$ offers a good opportunity to measure magnetic fields in carbon stars. First of all, because of the large abundance of the CN radical (up to $\sim 10^{-5}$, Bachiller et al. 1997a,b), the
$N=1 \rightarrow 0$ and $N=2 \rightarrow 1$ lines have already been observed and easily detected in several carbon-rich AGB stars and PNe (e.g., Bachiller et al. 1997a,b; Josselin \& Bachiller 2003). Moreover, $\mathrm{CN}$ is a paramagnetic species, thus exhibiting Zeeman splitting when the spectral line-forming region is permeated by a magnetic field $B$. The only currently viable technique for measuring the magnetic field strength in the circumstellar envelope of carbon stars is to detect the Zeeman effect in spectral lines excited in the envelope. The normal Zeeman effect splits a line with rest frequency $v_{0}$ into three separated polarised components with frequencies $v_{0}-v_{z}, v_{0}$ and $v_{0}+v z$, where $2 v_{z}=|B| Z$, where $Z$ is the Zeeman factor.

The $\mathrm{CN} \mathrm{N}=1-0$ line has a total of nine hyperfine components split into two groups (one around $113.17 \mathrm{GHz}$ and the second one around $113.49 \mathrm{GHz}$ ), with seven main lines, out of which four exhibit a strong Zeeman effect (see last column of Table 3, lines 1, 4, 5, and 7).

\subsection{Analysis method}

\subsubsection{Data reduction}

An electromagnetic wave is defined by its horizontal and vertical components:

$e_{\mathrm{H}}(z, t)=E_{\mathrm{H}} \mathrm{e}^{j(\omega t-k z-\delta)}$,

$e_{\mathrm{V}}(z, t)=E_{\mathrm{V}} \mathrm{e}^{j(\omega t-k z)}$,

where $\delta$ is the phase difference between horizontal and vertical components.

For each source observed in polarimetry at the $30 \mathrm{~m}$ and each VESPA section, that is, each CN hyperfine lines group (lines 1-3 and 4-7 in Table 3), the spectrometer output is converted to the Stokes parameters as defined in the equatorial reference frame (i.e. counting the polarisation angle from north to east):

$$
\begin{aligned}
I & =\left\langle E_{\mathrm{H}}{ }^{2}\right\rangle+\left\langle E_{\mathrm{V}}{ }^{2}\right\rangle \\
Q & =\left\langle E_{\mathrm{H}}{ }^{2}\right\rangle-\left\langle\left\langle E_{\mathrm{V}}{ }^{2}\right\rangle\right. \\
U & =2\left\langle E_{\mathrm{H}} E_{\mathrm{V}} \cos \delta\right\rangle \\
V & =2\left\langle E_{\mathrm{H}} E_{\mathrm{V}} \sin \delta\right\rangle .
\end{aligned}
$$

All data are reduced using the CLASS software ${ }^{1}$. All $I, Q, U$, and $V$ spectra have been inspected individually. A few of them have been discarded due to technical problems. A baseline has been removed from all $I, U, Q$, and $V$ spectra (excluding, in the $U$ and $V$ spectra, the frequency range where the $\mathrm{CN}$ line emission in Stokes $I$ is above the noise) using an order two polynomial.

\subsubsection{Numerical method for $V$ spectra}

In this subsection we use the Stokes $I$ and $V$ spectra obtained after data reduction, as shown in Fig. 1. To determine the magnetic field, Crutcher et al. (1996) developed a procedure to fit all seven hyperfine components in the Stokes $V$ spectra in the leastsquares sense:

$V_{i}(v)=C_{1} I_{i}(v)+C_{2} \frac{\mathrm{d} I_{i}(v)}{\mathrm{d} v}+C_{3} Z_{i} \frac{\mathrm{d} I_{i}(v)}{\mathrm{d} v}$,

with $i=1$ to 7 for the seven hyperfine components exhibiting a strong Zeeman effect.

In this expression $V_{i}(v)$ and $I_{i}(v)$ are the Stokes $V$ and $I$ spectra, respectively, for each of the seven hyperfine $\mathrm{CN}$ lines. This

1 http://www.iram.fr/IRAMFR/GILDAS/ 

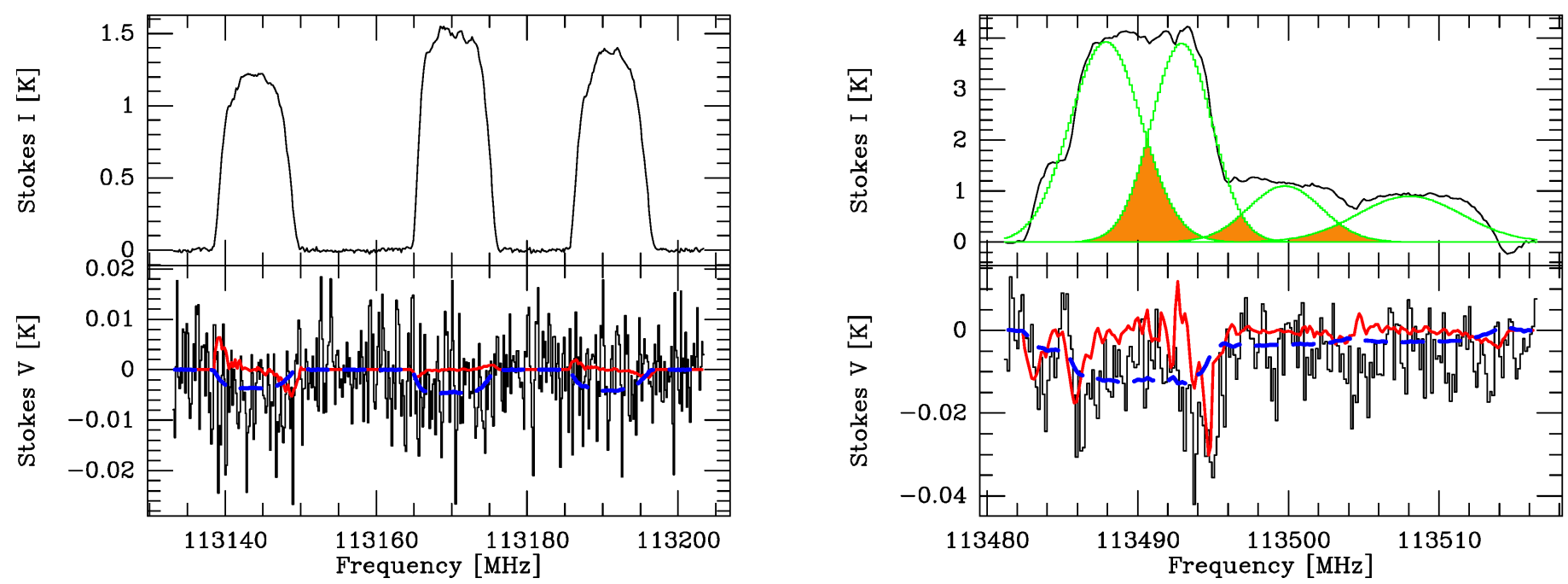

Fig. 1. IRC+10216: observations of November 2006 for the position $\left(-10^{\prime \prime},+20^{\prime \prime}\right)$. Top left: $\mathrm{CN}(1,1 / 2) \rightarrow(0,1 / 2)$ Stokes I spectrum. Bottom left: $V$ spectrum (in black) and least-squares fit (in red) using the Crutcher et al. (1996) method described in the text. The dashed blue line shows our fit assuming $C_{3}=0$. Right: same for the $\mathrm{CN}$ transition $(1,3 / 2) \rightarrow(0,1 / 2)$, with, in addition, the Gaussian fit for each individual line overplotted in green and the line overlap area coloured in orange on the $I$ spectrum.

method accounts for the Zeeman and instrumental effects. The instrumental contribution is determined by $C_{1}$ and $C_{2} . C_{1}$ depends on the gain difference in the telescope between the right (R) and left (L) circular polarisations. $C_{2}$ accounts for apparent Zeeman effect due to telescope beam squint when it observes sources with a velocity gradient or to residual instrumental effects leading to residual frequency offset between $\mathrm{R}$ and L. Hence, we can estimate the line of sight component of the magnetic field $\left(B_{\mathrm{los}}\right)$ from $C_{3}=\frac{B_{\mathrm{los}}}{2}$, once $C_{1}$ and $C_{2}$ have been calibrated.

We have developed a fitting procedure, applied simultaneously to the seven hyperfine components, which slightly differs from the "Crutcher technique" in that we do not fit for the three $C_{i}$ parameters simultaneously, but separately first. An initial estimated value is attributed to the parameters $C_{1}$ and $C_{2}$. In an iterative process, we then explore a large parameter space simultaneously for both $C_{1}$ and $C_{2}$ in order to get a rough estimate of the instrumental contribution. Next, still allowing $C_{1}$ and $C_{2}$ to vary (but within a smaller range of values), the parameter space for $\mathrm{C}_{3}$ is investigated (also in an iterative process) while $V$ is simultaneously calculated for the seven transitions. Convergence of the iterative process is checked by using a $\chi^{2}$ method and is reached when $0.18<\chi^{2}<0.23$ for our 2006 data and when $0.11<\chi^{2}<0.17$ for our 2016 data.

The main difficulty when using this method is to determine the frequency range corresponding to the emission of each $\mathrm{CN}$ hyperfine component. While the components of the first group (lines 1-3 in Table 3) are spectrally well identified (Fig. 1 left part), lines 4-7 are blended for the sources studied here (Fig. 1 right part). It is thus necessary to determine the frequency intervals where the overlap occurs. For these intervals, a blend of individual Stokes features is seen, that is, $V_{4}$ and $V_{5}$ for the $\mathrm{CN}$ hyperfine components 4 and 5, respectively:

$V_{4}+V_{5}=C_{3}\left(Z_{4} \frac{\mathrm{d} I_{i}(v)}{\mathrm{d} v}+Z_{5} \frac{\mathrm{d} I_{i}(v)}{\mathrm{d} v}\right)$

These overlapping frequencies (plotted in orange in Fig. 1, right part) are derived from a Gaussian fit (using CLASS) of the $\mathrm{CN}$ (1-0) lines profiles (see Fig. 1 right part, in green).

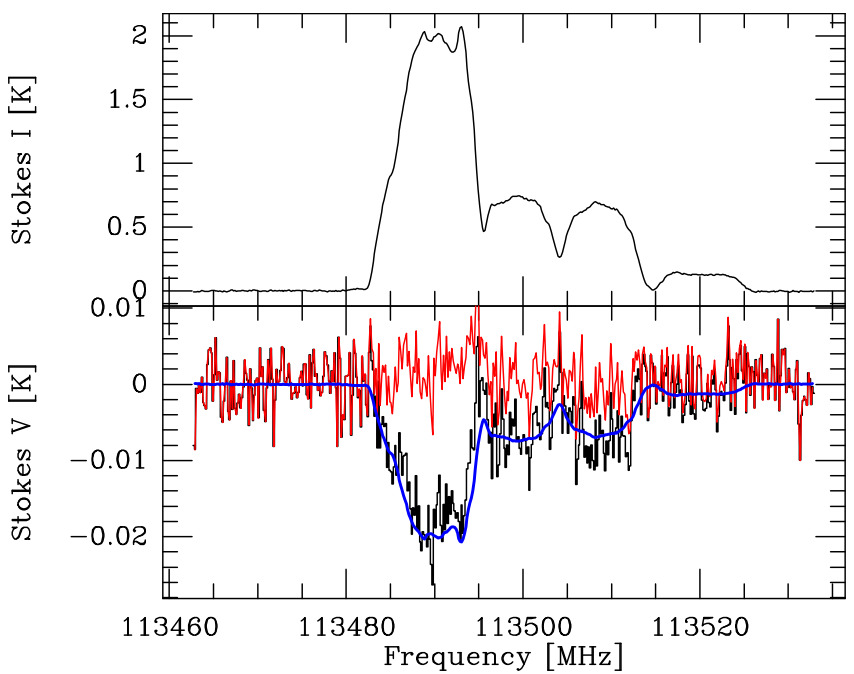

Fig. 2. IRC+10216: observations of March and June 2016 for position $\left(+20^{\prime \prime},+16^{\prime \prime}\right)$. CN $(1,3 / 2) \rightarrow(0,1 / 2)$ Stokes $I$ (top) and $V$ (bottom) spectra in black and fit of the estimation of the $I$ leakage in blue. The resulting true $V$ spectrum after removal of the $I$ leakage is shown in red.

\subsubsection{Specific treatment for the 2016 observations}

As explained in Sect. 3, in November 2015, the $3 \mathrm{~mm}$ mixer and the $30 \mathrm{~m}$ optics were modified. The dual-horn system was changed to a single horn system and the vertical (V) and horizontal $(\mathrm{H})$ polarisation splitting is produced now by an orthomode transducer. Using a single horn instead of two different horns for $\mathrm{H}$ and $\mathrm{V}$ should eliminate the misalignment of the $\mathrm{H}$ and $\mathrm{V}$ horns as a major contribution to the instrumental polarisation.

According to the IRAM technical tests (IRAM internal communication), the instrumental contamination in the linear polarisation is improved and the beam squint effect has been removed. However, our March 2016 observations showed that the $V$ instrumental polarisation has strongly increased. This change has produced a substantial leakage of the Stokes $I$ signal into the Stokes $V$, as revealed by the mirror image of $I$ seen in the $V$ spectra (see Fig. 2). 
We now consider the relative importance of the three $C_{i}$ parameters in formula (7). As the beam squint effect is now cancelled, we assume that the $C_{2}$ coefficient is equal to zero and that we are dominated by the leakage of the $I$ into $V$, that is, $C_{1}$. To estimate the leakage of $I$ into $V$, measured by the $C_{1}$ coefficient, we first assume that the magnetic field is negligible with respect to the leakage, that is, $C_{3}=0$. Hence, formula (7) becomes:

$V=V_{\mathrm{cal}_{i}}(v)=C_{1} I_{i}(v)$.

$C_{1}$ gives the percentage of the $I$ into $V$ leakage for the observations of IRC+10216 performed in March and June 2016. $C_{1}$ is found around 1.1 and $1.4 \%$ (see Table 4) depending on the observed positions. The resulting $V_{\text {cal }}$ spectra is shown in blue for one observed position in Fig. 2. No correlation has been found between $C_{1}$ and $I$ or the degree of elevation of the source.

Finally, to estimate the true $V$ signal ( $V_{\text {true }}$, i.e. not contaminated by $I$ ) we then subtract $V_{\text {cal }}$ from the original $V_{\text {ori }}$ spectra, that is to say:

$V_{\text {true }}=V_{\text {ori }}-V_{\mathrm{cal}_{i}}(v)=V_{i}-C_{1} I_{i}(v)$.

This leakage-free $V$ spectrum, $V_{\text {true }}$, is plotted in red in Fig. 2. Then, we can apply the analysis described in Sect. 4.2.2 on this $V_{\text {true }}$ signal, to derive the $C_{3}$ coefficient.

\section{Results}

\subsection{Uncertainties and method limitations}

As a consequence of formula (7), the measured accuracy of the magnetic field $\left(B_{\text {los }}\right)$ strength depends on 1$)$ the rms of the $I$ and $V$ observations and 2) the precise characterisation of the instrumental contamination.

\subsubsection{Zeeman features in the Stokes $V$ spectra}

For all sources/positions, the rms (for a velocity resolution of $0.2 \mathrm{~km} \mathrm{~s}^{-1}$ ) of the $I$ and $V$ observations is in the range $9-14 \mathrm{mK}$, except for RY Dra for which values are 4.5 times larger. The resulting $S / N$ (computed from the $V$ integrated area divided by rms $\times \delta v$ ) for the likely Zeeman features in the Stokes $V$ spectra is less than 3 for RW LMi, RY Dra, and NGC 7027, while it is more than 5 for AFGL618. Concerning IRC+10216, for five of the observed positions, the Zeeman effect is likely detected (considering the leakage-free $V_{\text {true }}$ spectrum for the 2016 data) with a $S / N$ higher than 5 (see Figs. 1, 2, 4, 5, and A.1-A.5). The two positions with the highest $S / N,\left(-10^{\prime \prime},+20^{\prime \prime}\right)$ and $\left(+20^{\prime \prime},+16^{\prime \prime}\right)$ are shown in Figs. 1 and 4. No signal is detected for the central position while the $S / N$ for position $\left(-18^{\prime \prime},-10^{\prime \prime}\right)$ is less than 3 . Only upper limits for $B$ will then be inferred when the $S / N$ is less than 3 .

\subsubsection{Accuracy of B-strength estimates}

As explained in Sect. 4.2, we have estimated, through an iterative process, the polarisation instrumental contributions from the total intensity, accounting for the leakage of $I$ into Stokes $V$, and from the beam squint effect for all observed sources/positions in our sample (see Tables 4 and 5). The beam squint leakage must be considered when horizontal and vertical polarisation signals are not collected in the same horn, that is, do not probe exactly the same region, and for sources with a non-zero velocity gradient. This effect, quantified by the $C_{2}$ coefficient, is noticeable in our first set of observations but has disappeared in the second

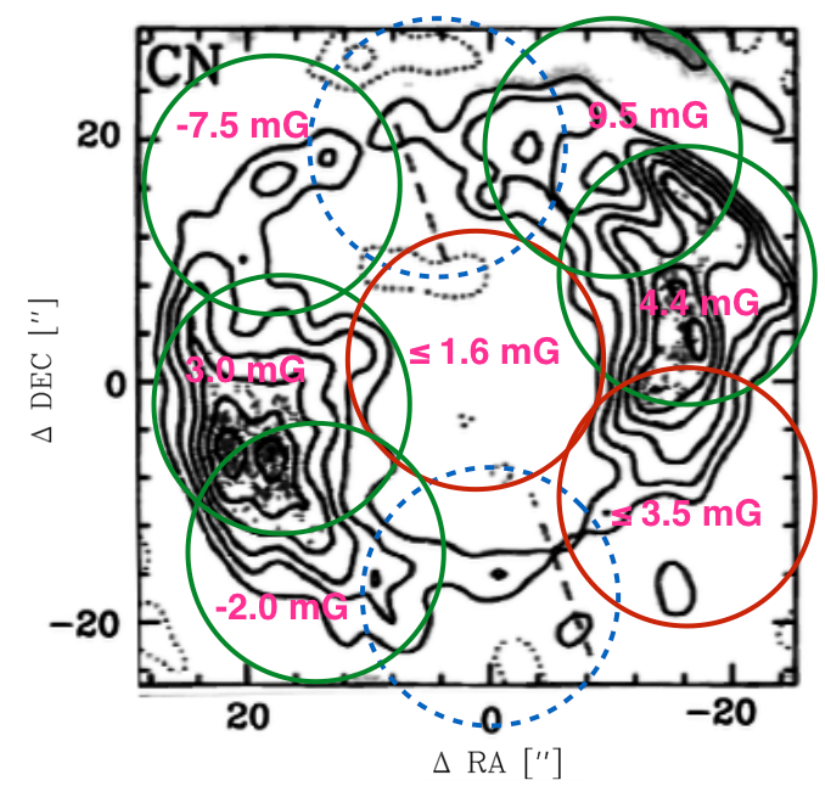

Fig. 3. CN map of IRC+10216 adapted from Lucas et al. (1995). Green, red, and dashed blue circles represent positions with a Zeeman detection, without a Zeeman detection, and unobserved positions, respectively. The diameter of the circles corresponds to the size of the telescope's primary beam $\left(21.7^{\prime \prime}\right)$.

run after a single horn has been installed on the 30-m telescope. Hence, while $C_{2}$ is zero for the March 2016 observations, we obtain large values for the other observations. The $C_{1}$ parameter varies between $5.5 \times 10^{-4}$ and $3.0 \times 10^{-3}$ for the first set of observations while it reaches a value of up to $1.4 \times 10^{-2}$ after the strong leakage of $I$ into $V$ occurs.

Considering the rms of the $\mathrm{V}$ spectra, we have estimated that the accuracy on our estimate of $C_{1}$ and $C_{2}$ is roughly $10 \%$ and $300 \mathrm{~Hz}$, respectively. From Eq. (7),

$C_{3} Z_{i} \frac{\mathrm{d} I_{i}(v)}{\mathrm{d} v}=V_{i}(v)-C_{1} I_{i}(v)-C_{2} \frac{\mathrm{d} I_{i}(v)}{\mathrm{d} v}$,

and, assuming that the error on $\mathrm{d} I_{i}(v) / \mathrm{d} v$ is negligible compared to the others, we can estimate the error on $C_{3}$ from:

$\delta\left(C_{3} Z_{i} \frac{\mathrm{d} I_{i}(v)}{\mathrm{d} v}\right)=\delta V_{i}(v)-\delta C_{1} I_{i}(v)-C_{1} \delta I_{i}(v)-\delta C_{2} \frac{\mathrm{d} I_{i}(v)}{\mathrm{d} v}$.

The error on the observed $V_{i}(v)$ and $I_{i}(v)$ being the spectral rms $\left(\mathrm{rms}_{V}\right.$ and $\left.\mathrm{rms}_{I}\right)$, one derives the uncertainty on $C_{3}$, hence on the magnetic field strength:

$\delta C_{3}=\frac{1}{Z_{i} \frac{\mathrm{d} I_{i}(v)}{\mathrm{d} v}} \times\left(\mathrm{rms}_{V}-\delta C_{1} I_{i}(v)-C_{1} \mathrm{rms}_{I}-\delta C_{2} \frac{\mathrm{d} I_{i}(v)}{\mathrm{d} v}\right)$

It is, in principle, possible to relate $C_{1}$ and $C_{2}$ to the Stokes $V$ and Stokes $I$ power patterns of the telescope. Thanks to the orthomode transducer, the power pattern for the instrumental conversion of Stokes $I$ into Stokes $V$ is almost axially symmetric. This justifies our assumption that $C_{2}$ is insignificant in our 2016 observations. A comparison between $C_{1}$ and the power patterns measured on Uranus can be found in Appendix B.

In addition, for the central position $\left(0^{\prime \prime}, 0^{\prime \prime}\right)$ of IRC +10216 , our spectra are slightly contaminated by the $\mathrm{CN}$ ring emission (see Fig. 3). A fraction of the signal is probably contaminated by the secondary telescope lobes located on the $\mathrm{CN}$ envelope (see Appendix B). 
Table 4. CN cartography of IRC +10216 .

\begin{tabular}{lccccc}
\hline \hline $\begin{array}{l}\text { Position } \\
\operatorname{arcsec}\end{array}$ & $\begin{array}{c}B_{\text {los }} \\
{[\mathrm{mG}]}\end{array}$ & $\begin{array}{c}\delta B_{\text {los }} \\
{[\mathrm{mG}]}\end{array}$ & $\begin{array}{c}\left|B_{r_{*}}\right| \\
{[\mathrm{G}]}\end{array}$ & $\begin{array}{c}\left|C_{1}\right| \\
\times 10^{-3}\end{array}$ & $\begin{array}{c}C_{2} \\
{[\mathrm{~Hz}]}\end{array}$ \\
\hline$+0+0^{a}$ & $\leq 1.6$ & & $\leq 1.1$ & 1.05 & 530 \\
$-10+20^{a}$ & 9.5 & 5.5 & 7.2 & 3.0 & 800 \\
$+15-15^{a}$ & -2.0 & 6.7 & 1.5 & 0.55 & 750 \\
\hline$-18+10$ & 4.4 & 1.8 & 3.3 & 12 & no \\
$-18-10$ & $\leq 3.5$ & & $\leq 2.7$ & 12 & no \\
$+18-04$ & 3.0 & 0.5 & 2.2 & 14 & no \\
$+20+16$ & -7.5 & 1.2 & 5.7 & 10 & no \\
\hline
\end{tabular}

Notes. For each position, $B_{r_{*}}$ is the extrapolated strength of the magnetic field (following a $1 / r$ law) at one stellar radius (the $\mathrm{CN}$ layer is at $2500 \mathrm{AU}$, i.e. $21^{\prime \prime}$, the stellar radius being $\left.3.3 \mathrm{AU}\right) .{ }^{(a)}$ Observations made in 2006 before the optics modification (see Sect. 4.2).

Of course, a strong limitation of these measurements is that the magnetic field strength is only measured along the line-ofsight. As a consequence, finding a zero magnetic field does not necessarily mean that there is no magnetic field; only the sum of the magnetic field vectors in the case of a twisted field could be zero within the telescope beam. Even if interferometric mapping of the Zeeman effect gave a higher angular resolution map of the line-of-sight component and then helped to constrain this possibility, it would not give us access to the full magnetic vector. To further investigate this issue, interferometric observations would nevertheless be helpful, but the spectral line polarimetry (with all Stokes parameters) is still not available with ALMA or NOEMA.

\subsection{Mapping the magnetic field in $I R C+10216$}

IRC+10216 is an AGB carbon star whose $\mathrm{CN}$ ring diameter is larger than the $30 \mathrm{~m}$ beam. For that reason, IRC+10216 is the best candidate to obtain, for the first time, a map of the magnetic field in the envelope of an evolved star. Nine positions have been proposed to cover the whole $\mathrm{CN}$ ring with half-beam spacing (see Fig. 3). Unfortunately, due to the weather and increasing observing time because of the Xpol issue (see Sect. 4.2.3), only seven out of the nine positions have been observed, three during the first run in 2006, and four in 2016. Results are presented in Table 4 (the CN cartography).

For the central position, we first note that the Stokes $I$ line profiles exhibit a double horn profile for each $\mathrm{CN}$ component (see Fig. 5), meaning that the $\mathrm{CN}$ is expanding. We measured an expansion velocity of about $14.0 \mathrm{~km} \mathrm{~s}^{-1}$, in agreement with Fong et al. (2006). On this central position (see Fig. 5) and on $\left(-18^{\prime \prime},-10^{\prime \prime}\right)$, the $V$ signal is dominated by the noise (see Sect. 5.1.1) and only upper limits for $B_{\text {los }}$ are derived. The other positions exhibit a likely Zeeman effect with a good $S / N$ (see Figs. 4-5). Considering the instrumental contribution, we then derive a longitudinal component (absolute value) of the magnetic field between 2.0 and $9.5 \mathrm{mG}$ depending on the position (see Table 4), with uncertainties varying from $15 \%$ (position $\left(+20^{\prime \prime},+16^{\prime \prime}\right)$ ) to $60 \%$ (position $\left(-10^{\prime \prime},+20^{\prime \prime}\right)$ ). The error on the measurement for positions $\left(+15^{\prime \prime},-15^{\prime \prime}\right)$ is more than three times the estimate of the $B_{\text {los }}$, which then should be considered as an order of magnitude estimate only. For two positions, the sign of the line-of-sight component of the vector $B$ is negative.

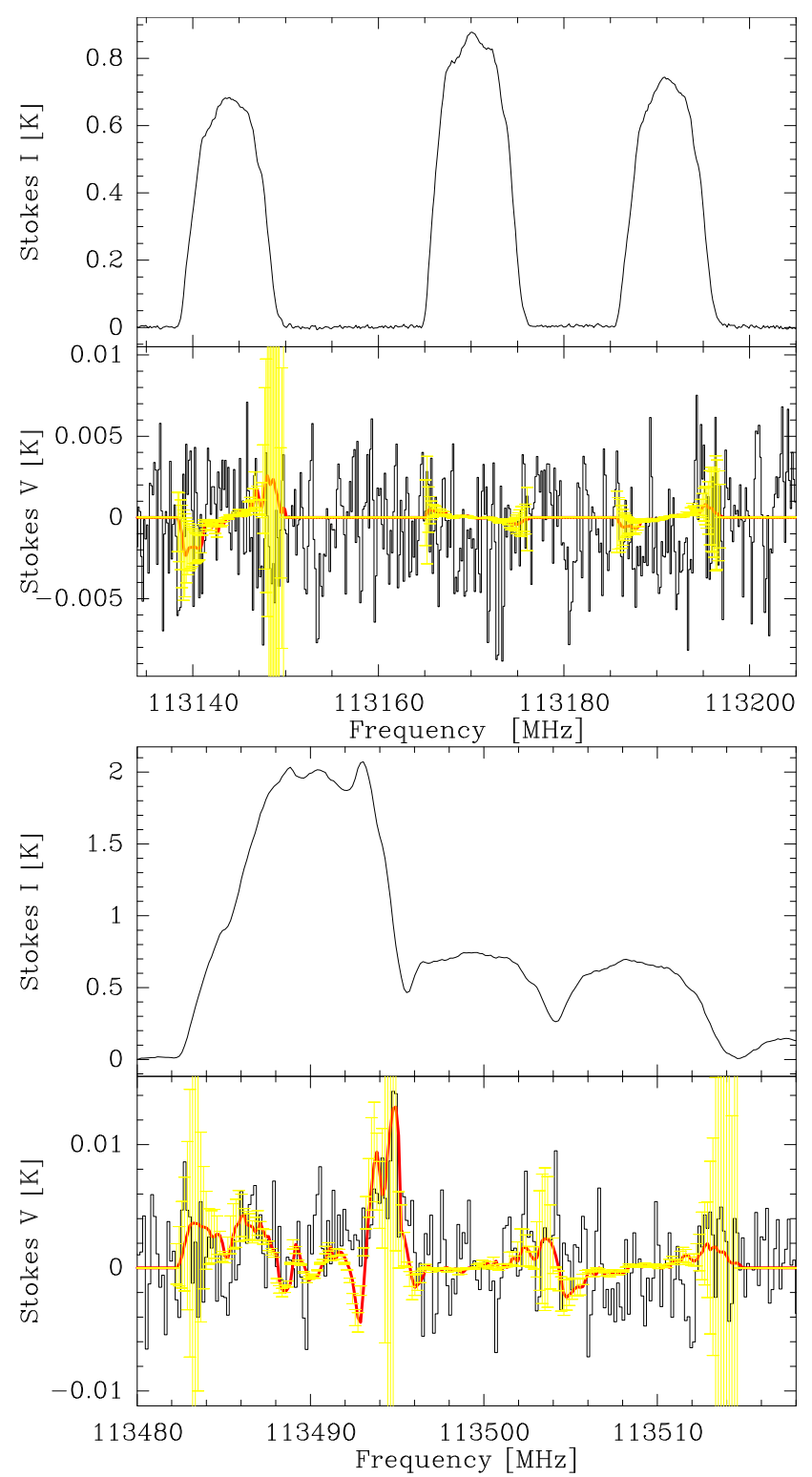

Fig. 4. IRC+10216: observations of March and June 2016 for the position $\left(+20^{\prime \prime},+16^{\prime \prime}\right)$. Top: stokes $I$ and $V$ for $\mathrm{CN}$ transition $(1,1 / 2) \rightarrow$ $(0,1 / 2)$. Bottom: same for $\mathrm{CN}$ transition $(1,3 / 2) \rightarrow(0,1 / 2)$. Spectra and least-squares fits for $V$ are shown in black and red, respectively. The error for the Stokes $V$ fit is plotted in yellow.

\subsection{Other objects}

Because of high noise, the observed Stokes $V$ signal (see Figs. A.6 and A.7) only allows us to derive an upper limit of the magnetic field $B_{\text {los }}$ along the line-of-sight for the two other AGB stars. We find $14.2 \mathrm{mG}$ and $3.8 \mathrm{mG}$ for RY Dra and RW LMi, respectively (see Table 5).

For the PPN AFGL618, the Stokes $V$ signal is detected above the instrumental contribution and the noise (see Fig. A.8 and Table 5). The magnetic field $B_{\mathrm{los}}$ is then estimated to be $6.0 \mathrm{mG}$ for this object. But again, considering the error found, we can only say that we have obtained an order of magnitude estimate of the magnetic field strength $B_{\text {los }}$. Concerning the young PN NGC 7027 (see Fig. A.9), an upper limit of $B_{\text {los }}$ is estimated to be $8 \mathrm{mG}$. 

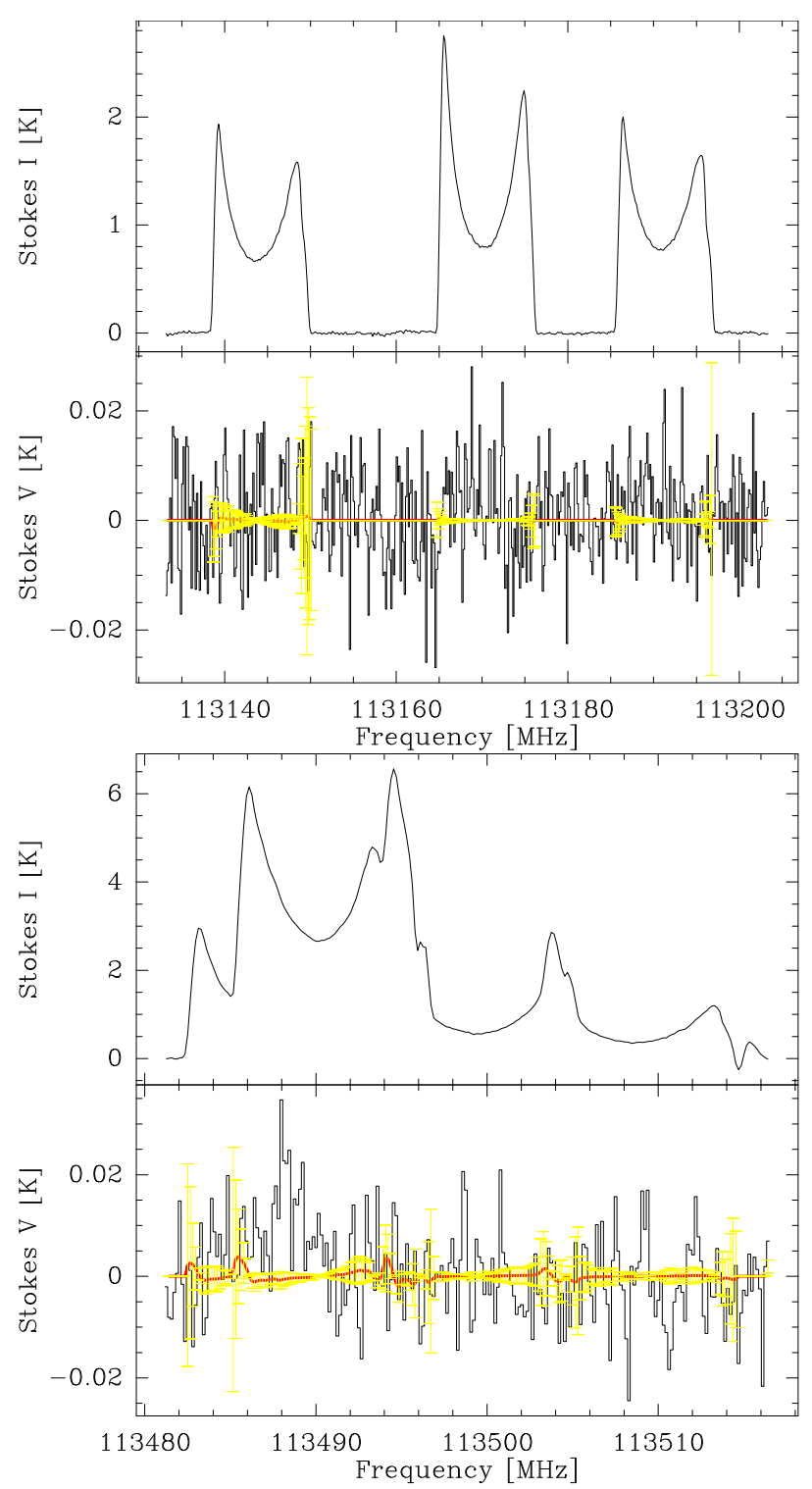

Fig. 5. As in Fig. 4 for observations of November 2006 for the position $\left(0^{\prime \prime}, 0^{\prime \prime}\right)$ toward IRC+10216.

Table 5. Estimated magnetic field strength on the line-of-sight $B_{\text {los }}$, its uncertainty, strength of the magnetic field extrapolated (following a $1 / r$ law) at one stellar radius (see Sect. 6) $B_{r_{*}}$, and instrumental contribution parameters $C_{1}$ and $C_{2}$ for each object in our sample.

\begin{tabular}{lccccc}
\hline \hline Object & $\begin{array}{c}B_{\text {los }} \\
{[\mathrm{mG}]}\end{array}$ & $\begin{array}{c}\delta B_{\text {los }} \\
{[\mathrm{mG}]}\end{array}$ & $\begin{array}{c}B_{r_{*}} \\
{[\mathrm{G}]}\end{array}$ & $\begin{array}{c}\left|C_{1}\right| \\
\times 10^{-3}\end{array}$ & $\begin{array}{c}C_{2} \\
{[\mathrm{~Hz}]}\end{array}$ \\
\hline RW LMi & $\leq 3.8$ & & $\leq 4.4$ & 1.05 & 9200 \\
RY Dra & $\leq 14.2$ & & $\leq 4.8$ & 1.15 & 17500 \\
AFGL618 & 6.0 & 6.0 & 67.5 & 1.05 & 2750 \\
NGC 7027 & $\leq 8.0$ & & $\leq 3.1 \times 10^{5}$ & 1.1 & 1360 \\
\hline
\end{tabular}

\section{Discussion}

\subsection{Distribution of the magnetic field in $I R C+10216$}

Considering the symmetry of the $\mathrm{CN}$ ring in Fig. 3 (see Lucas et al. 1995), and assuming that the magnetic field should be stronger where the $\mathrm{CN}$ material is denser, we should expect similar values of the magnetic field for different pairs of positions: $\left(-10^{\prime \prime},+20^{\prime \prime}\right) /\left(+15^{\prime \prime},-15^{\prime \prime}\right),\left(+20^{\prime \prime},+16^{\prime \prime}\right) /\left(-18^{\prime \prime},-10^{\prime \prime}\right)$, and $\left(+18^{\prime \prime},-04^{\prime \prime}\right) /\left(-18^{\prime \prime},+10^{\prime \prime}\right)$. This is not the case, however. We observe a stronger magnetic field in the northern part of the ring where $\mathrm{CN}$ seems to be less dense. Furthermore, we could also expect a stronger magnetic field for positions $\left(+18^{\prime \prime},-04^{\prime \prime}\right)$ and $\left(-18^{\prime \prime},+10^{\prime \prime}\right)$ where $\mathrm{CN}$ is observed to be more intense, but this is not the case either, even though position $\left(-18^{\prime \prime},+10^{\prime \prime}\right)$ overlaps region $\left(-10^{\prime \prime},+20^{\prime \prime}\right)$ where $B$ is strong. The non-detection of the Zeeman effect for position $\left(+0^{\prime \prime},+0^{\prime \prime}\right)$ is consistent with the $\mathrm{CN}$ hole and with oppositely oriented magnetic field vectors in front and near-side (Fig. 3).

There are several possible explanations for the nondetections in our observations: (1) $\mathrm{CN}$ is less abundant at some positions; (2) the magnetic field vectors cancel out when averaged within the beam; (3) the magnetic field distribution is not homogeneous.

First of all, the total integrated $\mathrm{CN}$ intensities for each position show that the $\mathrm{CN}$ distribution has slightly changed since the observations of Lucas et al. (1995): the western part of the CN ring is now the weakest one while the emission coming from the northern part is now stronger. Nevertheless, there is no obvious correlation between the field strength $B_{\mathrm{los}}$ and $\mathrm{CN}$ emission.

From this Zeeman effect study we have also inferred the sign, that is, the direction, of the line-of-sight component of the magnetic field vector, which is negative for positions $\left(+15^{\prime \prime},-15^{\prime \prime}\right)$ and $\left(+20^{\prime \prime},+16^{\prime \prime}\right)$ and positive for all other positions. As a consequence, no obvious direction pattern is observed. We underline that a magnetic field perpendicular to the observed $\mathrm{CN}$ ring would cause the same sign everywhere while a toroidal field within the mapped $\mathrm{CN}$ ring, with the torus slightly inclined, would produce a characteristic $B_{\text {los }}$ distribution: zero at the centre and at the minor axis positions while maximum at the major axis positions with sign reversed from one side to the other.

Men'shchikov et al. (2001) modelled the geometrical structure of the envelope within three regions: the inner most dense core with bipolar cavities (outflow) over 60 AU ( $\left.0^{\prime \prime} 5\right)$, a lessdense envelope where molecules are observed, and the outer extended envelope $\left(6 \times 10^{5} \mathrm{AU} \sim 1^{\circ} 3\right)$. The opening angle of the cavities is $36^{\circ}$ and the viewing angle between the equatorial plane and the line of sight is $40^{\circ}$. Therefore, we can extrapolate that the less dense northern and southern parts of the $\mathrm{CN}$ ring in the Lucas et al. (1995) map correspond to the continuity of the cavities. As a consequence, since 1995, the $\mathrm{CN}$ ring might have been modified by a change in the cavities or the viewing angle has changed (unlikely). Moreover, the measured strength of the magnetic field $B_{\text {los }}$ could depend on the viewing angle of the outflow cavities assuming that magnetic field vectors follow the outflow cavities.

\subsection{Comparison with other observations and implications for the magnetic field mechanism}

We tried to verify that our magnetic field estimates are consistent with previous studies to date exclusively dedicated to $\mathrm{O}$ rich stars. We now intend to link these results to other detections in the O-rich stellar environments. Assuming that the magnetic field process does not depend on the chemical type of the star, and knowing that the $\mathrm{CN}$ layer for $\mathrm{C}$-rich objects is roughly at the same distance as the $\mathrm{OH}$ layer for O-rich objects, we can compare the values of $B$ for these two layers. These results (see Tables 4 and 5) are compatible with an estimate of the $B_{\text {los }}$ field from $\mathrm{OH}$ masers observed for instance by Rudnitski et al. (2010) or Gonidakis et al. (2014). 


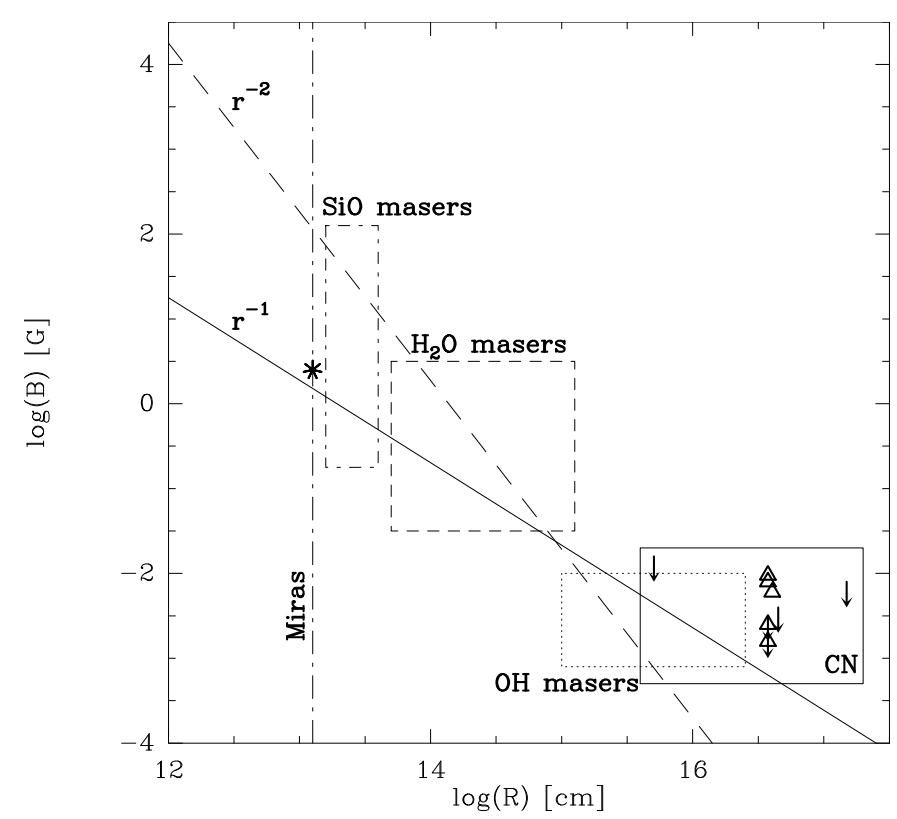

Fig. 6. Magnetic field strength as a function of the distance (Vlemmings 2012). The different boxes show the range of observed magnetic field strengths derived from observations of $\mathrm{SiO}$ masers (Kemball et al. 2009; Herpin et al. 2006), $\mathrm{H}_{2} \mathrm{O}$ masers (Vlemmings et al. 2002 and Vlemmings et al. 2005), OH masers (e.g Rudnitski et al. 2010) and CN (this work, triangles indicate Zeeman detection and arrows indicate the upper values). The dashed and solid lines indicate $r^{-2}$ solar-type and $r^{-1}$ toroidal magnetic field configurations, respectively. The vertical dashed line indicates the stellar surface for the Miras, and the star corresponds to the magnetic field measured at the surface of $\chi$ Cyg by Lèbre et al. (2014) for this object.

Earlier field measurements have shown that the magnetic field strength decreases across the envelope either in $1 / r$ or in $1 / r^{2}$ (Vlemmings 2012). This is shown in Fig. 6. Meanwhile, the first estimate of the magnetic field strength $(2-3 \mathrm{G})$ at the surface of a Mira star ( $\chi$ Cyg, $R_{\star}=2$ AU, Lacour et al. 2009) has been obtained by Lèbre et al. (2014) on Lacour et al. ( $\chi$ Cyg, $\left.R_{\star}=2 \mathrm{AU}, 2009\right)$. Plotted in Fig. 6, it strongly suggests an $1 / r$ variation. In order to verify this behaviour, we place our $B_{\text {los }}$ estimates for all stars in our sample (AGB, PPN and PN) in Fig. 6, which represents the measured magnetic field strength $B_{\text {los }}$ versus the radial distance from the centre of the star. Our results for C-rich, evolved objects confirm that a $1 / r$ variation is the most reliable scenario (and definitely exclude a variation in $1 / r^{3}$ ). All measurements together hence tend to favour a $1 / r$ variation of a toroidal magnetic field (as proposed by Pascoli 1997).

Adopting this law, we have thus extrapolated the $B$ field to a distance of one stellar radius $\left(B_{r_{*}}\right.$, see Tables 4 and 5). For the AGB stars RY Dra and RW LMi, the upper value for the magnetic field strength is of a few Gauss, while for IRC +10216 , the estimated surface magnetic field can be as high as $7.2 \mathrm{G}$ with an average value of $3.8 \mathrm{G}$. These values are in agreement with the estimate of Lèbre et al. (2014) for $\chi$ Cyg. For the PPN AFGL618, we derive a surface field of tens of Gauss. In this object, Sabin et al. (2014) have observed a well aligned and organised field along the polar direction (but no toroidal equatorial field) parallel to the major axis of the outflow. Our upper value to $B_{\text {los }}$ in the envelope of the PN NGC 7027 is compatible with Sabin et al. (2007) (a few mG at 3000-4000 AU) or Gómez et al. (2009) for the young PN K 3-35 (0.9 mG, based on $\mathrm{OH}$ masers). In fact, the estimate by Sabin et al. (2007) at 3000-4000 AU from polarimetric SCUBA observations implies that the magnetic field in the $\mathrm{CN}$ layer (10000 AU) should be weaker, thus explaining why it is not detected in our study. Moreover, beyond $5000 \mathrm{AU}$, according to these authors, there is no sign of an organised field, and, as a consequence, the line-ofsight $B_{\text {los }}$ that we measure here might be zero. Nevertheless, the clear correlation observed by these authors between the field orientation and the nebular structure at 3000-4000 AU underlines the importance of the magnetic field in this object. The magnetic field in AFGL618 and NGC 7027, with a strength of a few $\mathrm{mG}$, is dominant over the thermal pressure and drives a magneticoutflow-launching mechanism.

For the PN, the derived upper value of $B_{r_{*}}$ seems to be far too large compared to previous studies. In fact, several studies (e.g. Steffen et al. 2014) indicate an absence of a strong (kGauss) magnetic field at the PN stellar surface (of the central object), thus in contradiction with our own estimate assuming $1 / r$ law. While the stellar radius for AFGL618 (see Sánchez Contreras et al. 2002) is not well constrained, thus the $B_{r_{*}}$ estimate at the stellar surface remains uncertain, Latter et al. (2000) have estimated the radius of the central object of NGC 7027 at $3.5 \times 10^{-4}$ AU. We can therefore conclude that the magnetic field in the proto-PN and PN does not follow an $1 / r$ law or that estimates of the stellar radius for these objects is wrong.

Interpreting IRC +10216 is of course difficult because, as the source is resolved, we have different $B$ estimates. While the nondetection at the central position could be explained by a lower $\mathrm{CN}$ abundance, hence an overly weak signal, we should expect the same $B_{\text {los }}$ field in the two other observed NW and SE positions. On the contrary, only the NW position exhibits a clear Zeeman detection, hence a detectable $B_{\text {los }}$ field. This tends to show that the $B_{\text {los }}$ field is not homogeneously strong or aligned in the envelope. This result agrees with the study of the magnetic field using molecules CO, SIS, and CS by Girart et al. (2012), which suggests that the magnetic field morphology is possibly complex (the positions they studied are within the $\mathrm{CN}$ ring). A more detailed interferometric map of the magnetic field is mandatory to make any substantial conclusions, especially as a new spiral structure was recently discovered (Cernicharo et al. 2015).

\subsection{Impact on the stellar evolution}

The previous section has shown that a $r^{-1}$ decline of the magnetic field across the envelope of $\mathrm{O}$ - and $\mathrm{C}$-rich evolved objects is the most likely scenario (except for the PN object in our sample). As a consequence, the magnetic field appears to be toroidal in these evolved objects, and its strength is expected to be of a few Gauss at the stellar surface. This is in agreement with, for instance, the torus models of García-Segura \& López (2000). Nevertheless, our estimates of the magnetic field at the stellar surface, combined with the measurement of Lèbre et al. (2014) towards the S-type Mira star $\chi$ Cyg, are lower (except for AFGL618) than the prediction from Pascoli \& Lahoche (2010) of a 10-100 G surface field for an AGB star decreasing as $1 / r$. Compared to the field strength required for a toroidal field to launch an outflow via a field pressure gradient $(40 \mathrm{G}$ at $R_{\star}$ García-Segura et al. 2005), the magnetic field, as estimated here, is again too weak at the stellar surface. On the other hand, in the hybrid MHD dust-driven wind model for Mira of Thirumalai \& Heyl (2012) the role of a surface field of $\sim 4 \mathrm{G}$ is dynamically important in the star's mass-loss process. Moreover, Vlemmings (2011) has shown, based on the measured magnetic field found in the literature in $\mathrm{SiO}$ and $\mathrm{H}_{2} \mathrm{O}$, that the magnetic 
field dominates at and close to the photosphere. This is not the case in the $\mathrm{OH} / \mathrm{CN}$ region, or at least the field energy is comparable to the kinetic energy.

\section{Conclusion}

Using the polarimeter Xpol with the IRAM 30 m, we have made a study of the magnetic field in a small but representative sample of C-rich evolved stars (three AGB stars including the prototypical AGB IRC+10216, one PPN, and one PN). Thanks to the Zeeman effect in the CN 1-0 transition, we have been able to determine the magnetic field strength using Crutcher's hyperfine lines fitting method. The $\mathrm{CN}$ ring observed towards IRC +10216 is well resolved by the $30 \mathrm{~m}$ beam and we thus have been able to trace the magnetic field strength in the circumstellar envelope.

This work is the first estimate (apart from a preliminary announcement made by Herpin et al. 2009) of the magnetic field strength in the circumstellar envelope of C-rich objects. The hyperfine transitions of the $\mathrm{CN} \mathrm{N}=1-0$ transition were used to probe the field in these stars and, for the first time, to map the field distribution in the peculiar object IRC +10216 .

For AGB stars, we estimate the magnetic field in the CSE to be between 1.5 and $9.5 \mathrm{mG}$. Previous studies for O-rich evolved stars have shown that the magnetic field decreases across the CSE either in $1 / r$ or in $1 / r^{2}$ (where $r$ is the distance to the star's centre), with a preference for $1 / r$. Considering the magnetic field strength derived in the envelope of the objects studied here and the inferred value $B_{r_{*}}$ at the stellar surface (between 1.1 and $9.5 \mathrm{G}$ ), we conclude that the $B$ field varies in $1 / r$, as expected for a toroidal magnetic field. We stress that such a magnetic field is too weak to launch an outflow via a field pressure gradient. However, a surface magnetic field of a few Gauss may play an important role in the star's mass-loss process. Moreover, our map of IRC +10216 shows that the magnetic field is not homogeneously strong or aligned in the envelope and that the $\mathrm{CN}$ morphology might have changed between 1995 and now.

For the central stars of the proto-PN, AFGL618 and PN NGC 7027, we found $B_{\text {los }}=6.0 \mathrm{mG}$ and $B_{\text {los }} \leq 8.0 \mathrm{mG}$, respectively, corresponding to an improbably high surface magnetic field of $67 \mathrm{G}$ for AGL618 and an upper limit of $3.4 \times 10^{5} \mathrm{G}$ for NGC 7027 if $B$ varies in $1 / r$. For proto-PN and PN, we conclude that the magnetic field might not follow the $1 / r$ law, that is, something in the stellar evolution between AGB and post-AGB may have changed the field topology. More dedicated polarimetric observations in this class of objects are necessary.

We have carefully estimated the instrumental contamination in our study. Moreover, considering that we only measure the magnetic field along the line-of-sight, we stress that a nodetection does not necessarily imply that there is no magnetic field. Spectropolarimetric mapping using interferometers like ALMA and NOEMA are required to minimise this problem and to better understand the role of the magnetic field in the evolution of evolved stars.

Acknowledgements. We would like to thank the anonymous referee for his very useful and constructive comments. We also thank C. Kramer (IRAM-Granada) for his help in the understanding of the leakage issue with XPol.

\section{References}

Akashi, M., Sabach, E., Yogev, O., \& Soker, N. 2015, MNRAS, 453, 2115 Alcolea, J., Neri, R., \& Bujarrabal, V. 2007, A\&A, 468, L41

Assaf, K. A., Diamond, P. J., Richards, A. M. S., \& Gray, M. D. 2013, MNRAS, 431,1077
Aurière, M., Konstantinova-Antova, R., Charbonnel, C., et al. 2015, A\&A, 574, A90

Bachiller, R., Forveille, T., Huggins, P. J., \& Cox, P. 1997a, A\&A, 324, 1123

Bachiller, R., Fuente, A., Bujarrabal, V., et al. 1997b, A\&A, 319, 235

Balick, B., \& Franck, A. 2002, ARA\&A, 40, 439

Balick, B., Huarte-Espinosa, M., Frank, A., et al. 2013, ApJ, 772, 20

Blackman, E. G. 2009, in IAU Symposium, eds. K. G. Strassmeier, A. G. Kosovichev, \& J. E. Beckman, 259, 35

Boffin, H. M. J., Miszalski, B., Rauch, T., et al. 2012, Science, 338, 773

Bujarrabal, V., \& Alcolea, J. 2013, A\&A, 552, A116

Cernicharo, J., Marcelino, N., Agúndez, M., \& Guélin, M. 2015, A\&A, 575, A91

Chau, W., Zhang, Y., Nakashima, J.-I., Deguchi, S., \& Kwok, S. 2012, ApJ, 760, 66

Crutcher, R. M., Troland, T. H., Lazareff, B., \& Kazès, I. 1996, ApJ, 456, 217

De Beck, E., Decin, L., de Koter, A., et al. 2010, A\&A, 523, A18

De Beck, E., Lombaert, R., Agúndez, M., et al. 2012, A\&A, 539, A108

Decin, L., Richards, A. M. S., Neufeld, D., et al. 2015, A\&A, 574, A5

Desmurs, J. F., Bujarrabal, V., Colomer, F., \& Alcolea, J. 2000, A\&A, 360, 189

Fong, D., Meixner, M., Sutton, E. C., Zalucha, A., \& Welch, W. J. 2006, ApJ, 652,1626

García-Segura, G., \& López, J. A. 2000, ApJ, 544, 336

García-Segura, G., López, J. A., \& Franco, J. 2005, ApJ, 618, 919

Girart, J., Patel, N., Vlemmings, W., \& Rao, R. 2012, ApJ, 751, L20

Gómez, Y., Tafoya, D., Anglada, G., et al. 2009, ApJ, 695, 930

Gonidakis, I., Chapman, J. M., Deacon, R. M., \& Green, A. J. 2014, MNRAS, 443, 3819

Habing, H. J. 1996, A\&ARv, 7, 97

Herpin, F., Goicoechea, J. R., \& Cernicharo, J. 2002, ApJ, 577, 961

Herpin, F., Baudry, A., Thum, C., Morris, D., \& Wiesemeyer, H. 2006, A\&A, 450,667

Herpin, F., Baudry, A., Josselin, E., Thum, C., \& Wiesemeyer, W. 2009, in Cosmic Magnetic Fields From Planets, IAU Symp., 259, 47

Höfner, S., Bladh, S., Aringer, B., \& Ahuja, R. 2016, A\&A, 594, A108

Houde, M. 2014, ApJ, 795, 27

Huang, Z.-Y., Hasegawa, T. I., Dinh-V-Trung, et al. 2010, ApJ, 722, 273

Huggins, P. J., \& Glassgold, A. E. 1982, AJ, 87, 1828

Izumiura, H., \& Hashimoto, O. 1999, in Asymptotic Giant Branch Stars, eds. T. Le Bertre, A. Lebre, \& C. Waelkens, IAU Symp.,191, 401

Jordan, S., Bagnulo, S., Werner, K., \& O’Toole, S. J. 2012, A\&A, 542, A64

Josselin, E., \& Bachiller, R. 2003, ApJS, 397, 659

Kemball, A. J., \& Diamond, P. J. 1997, ApJ, 481, L111

Kemball, A. J., Diamond, P. J., Gonidakis, I., et al. 2009, ApJ, 698, 1721

Kim, H., Hsieh, I.-T., Liu, S.-Y., \& Taam, R. E. 2013, ApJ, 776, 86

Kim, H., Lee, H.-G., Mauron, N., \& Chu, Y.-H. 2015, ApJ, 804, L10

Konstantinova-Antova, R., Aurière, M., Charbonnel, C., et al. 2014, in IAU Symp., eds. P. Petit, M. Jardine, \& H. C. Spruit, 302, 373

Kwok, S., \& Bignell, R. C. 1984, ApJ, 276, 544

Lacour, S., Thiébaut, E., Perrin, G., et al. 2009, ApJ, 707, 632

Latter, W. B., Dayal, A., Bieging, J. H., et al. 2000, ApJ, 539, 783

Leal-Ferreira, M. L., Vlemmings, W. H. T., Diamond, P. J., et al. 2012, A\&A, 540, A42

Leal-Ferreira, M. L., Vlemmiings, W. H. T., Kemball, A., \& Amiri, N. 2013, A\&A, 554, A134

Lèbre, A., Aurière, M., Fabas, N., et al. 2014, A\&A, 561, A85

Lee, C.-F., Sahai, R., Sánchez Contreras, C., Huang, P.-S., \& Hao Tay, J. J. 2013, ApJ, 777, 37

Leone, F., Corradi, R. L. M., Martínez González, M. J., Asensio Ramos, A., \& Manso Sainz, R. 2014, A\&A, 563, A43

Lindqvist, M., Schöier, F. L., Lucas, R., \& Olofsson, H. 2000, A\&A, 361, 1036 Lucas, R., Guelin, M., Kahane, C., Audinos, P., \& Cernicharo, J. 1995, Ap\&SS, 224, 293

Masson, C. R. 1989, ApJ, 336, 294

Men'shchikov, A. B., Balega, Y., Blöcker, T., Osterbart, R., \& Weigelt, G. 2001, A\&A, 368, 497

Nordhaus, J., Blackman, E. G., \& Frank, A. 2007, MNRAS, 376, 599

Nyman, L.-A., Hall, P. J., \& Olofsson, H. 1998, A\&AS, 127, 185

Pascoli, G. 1997, ApJ, 489, 946

Pascoli, G., \& Lahoche, L. 2008, PASP, 120, 1267

Pascoli, G., \& Lahoche, L. 2010, PASP, 122, 1334

Ragland, S., Traub, W. A., Berger, J.-P., et al. 2006, ApJ, 652, 650

Ramstedt, S., \& Olofsson, H. 2014, A\&A, 566, A145

Rudnitski, G. M., Pashchenko, M. I., \& Colom, P. 2010, Astron. Rep., 54, 400

Sabin, L., Zijlstra, A. A., \& Greaves, J. S. 2007, MNRAS, 376, 378

Sabin, L., Zhang, Q., Zijlstra, A. A., et al. 2014, MNRAS, 438, 1794

Sabin, L., Wade, G. A., \& Lèbre, A. 2015, MNRAS, 446, 1988

Sánchez Contreras, C., Sahai, R., \& Gil de Paz, A. 2002, ApJ, 578, 269 
A. Duthu et al.: Magnetic field in IRC+10216 and other C-rich evolved stars

Schmidt, G. D., Hines, D. C., \& Swift, S. 2002, ApJ, 576, 429

Skinner, C. J., Meixner, M., \& Bobrowsky, M. 1998, MNRAS, 300, L29

Soker, N. 2000, ApJ, 540, 436

Steffen, M., Hubrig, S., Todt, H., et al. 2014, A\&A, 570, A88

Thirumalai, A., \& Heyl, J. S. 2012, MNRAS, 422, 1272

Thum, C., Wiesemeyer, H., Paubert, G., Navarro, S., \& Morris, D. 2008, PASP, 120,777

Tuthill, P. G., Monnier, J. D., Danchi, W. C., \& Lopez, B. 2000, ApJ, 543, 284

Vlemmings, W. H. T. 2011, in Asymmetric Planetary Nebulae 5 Conference, 89
Vlemmings, W. H. T. 2012, in Cosmic Masers - from OH to H0, eds. R. S. Booth, W. H. T. Vlemmings, \& E. M. L. Humphreys, IAU Symp., 287, 31

Vlemmings, W. H. T., Diamond, P., \& van Langevelde, H. J. 2001, A\&A, 375, $6 \mathrm{~L} 1$

Vlemmings, W. H. T., Diamond, P., \& van Langevelde, H. J. 2002, A\&A, 394, 589

Vlemmings, W. H. T., van Langevelde, H. J., \& Diamond, P. 2005, A\&A, 434, 1029

Western, L. R., \& Watson, W. D. 1983, ApJ, 275, 195 


\section{Appendix A: Figures}

Figures A.1 to A.9 show the stokes $I$ and $V$ spectra for all stars in our sample.

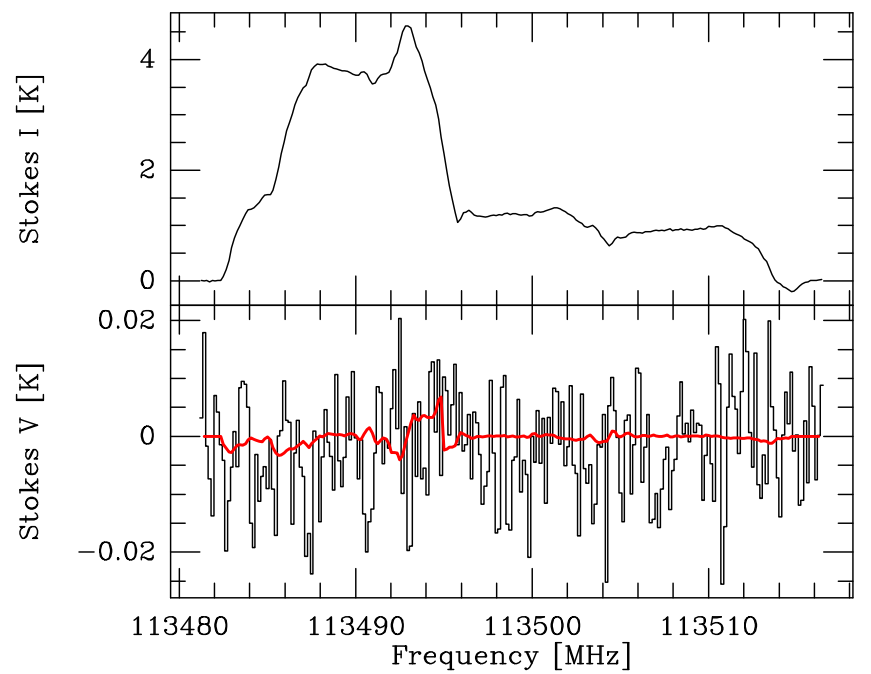

Fig. A.1. IRC+10216: observations of November 2006 for the position $\left(+15^{\prime \prime},-15^{\prime \prime}\right)$ and the $\mathrm{CN}$ transition $(1,3 / 2) \rightarrow(0,1 / 2)$. Top: Stokes $I$ spectrum. Bottom: spectra and least-squares fits to $V$ are shown in black and red, respectively.

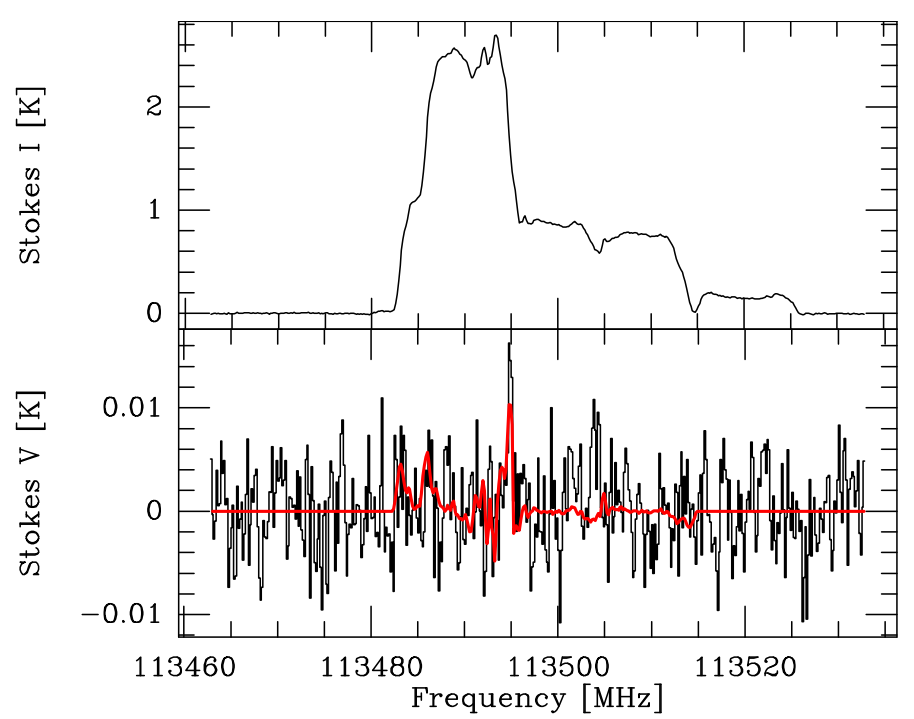

Fig. A.2. As in Fig. A.1 but for the observations of March and June 2016 toward position $\left(-18^{\prime \prime},+10^{\prime \prime}\right)$ of IRC +10216 .

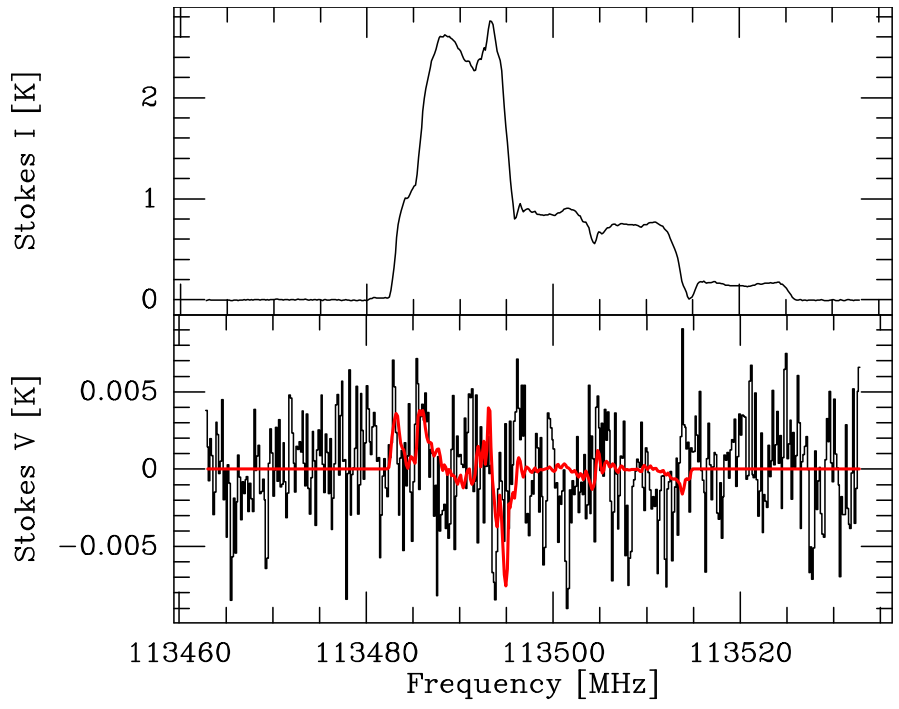

Fig. A.3. As in Fig. A.1 but for the observations of March and June 2016 toward position $\left(-18^{\prime \prime},-10^{\prime \prime}\right)$ of IRC +10216 .

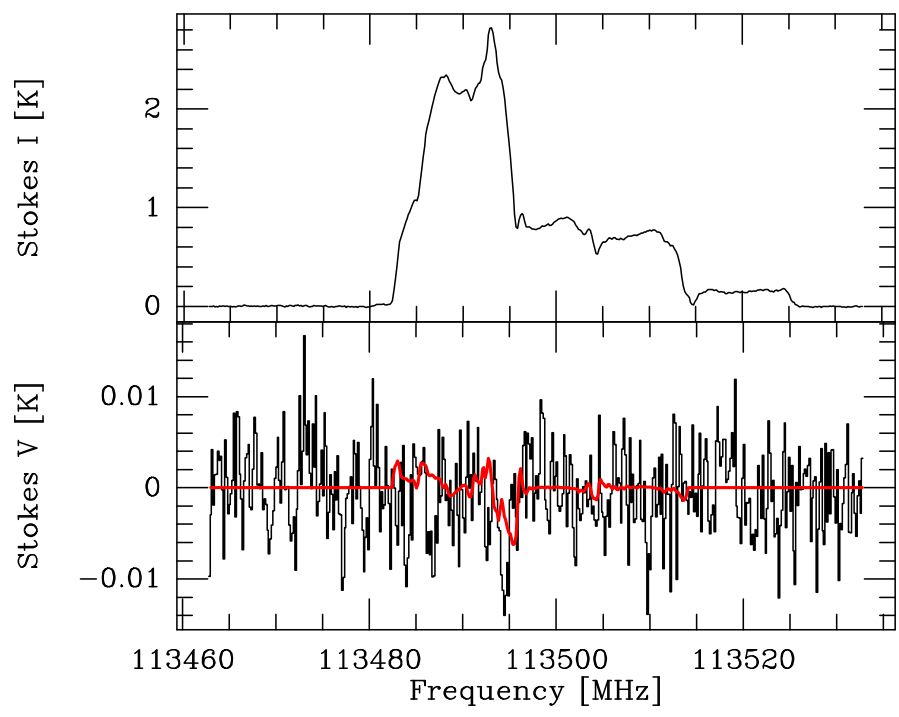

Fig. A.4. As in Fig. A.1 but for the observations of March 2016 toward position $\left(+18^{\prime \prime},-04^{\prime \prime}\right)$ of IRC +10216 .

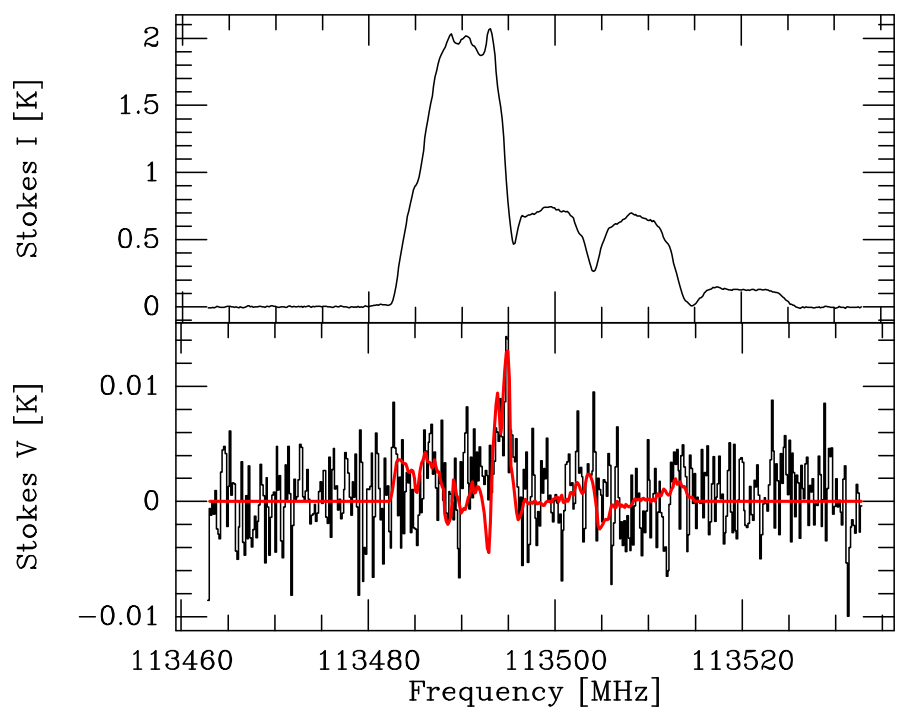

Fig. A.5. As in Fig. A.1 but for the observations of March and June 2016 toward position $\left(+20^{\prime \prime},+16^{\prime \prime}\right)$ of IRC +10216 . 
A. Duthu et al.: Magnetic field in IRC+10216 and other C-rich evolved stars

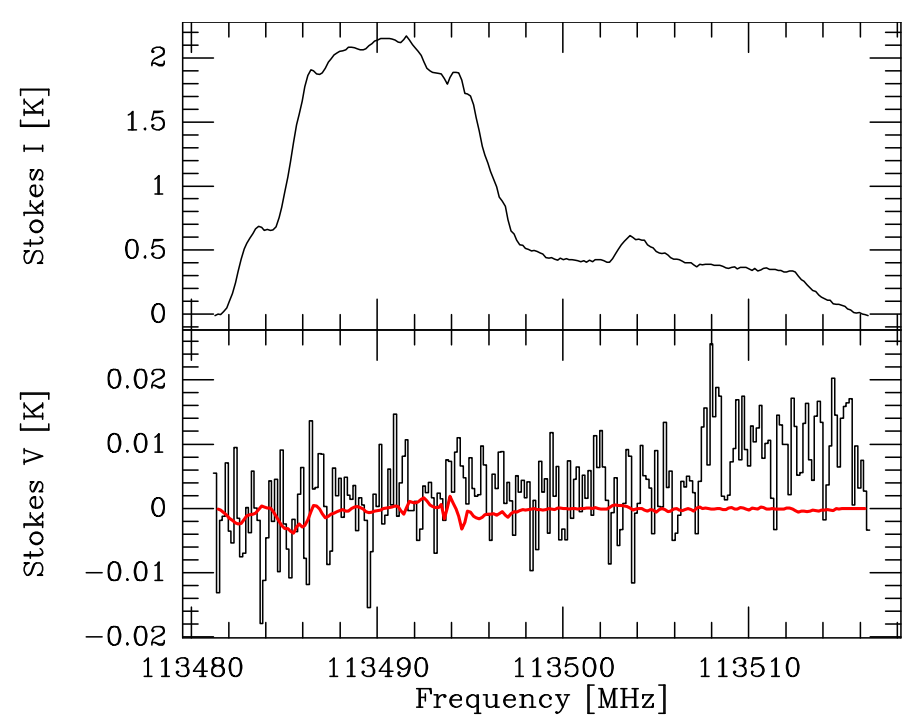

Fig. A.6. As in Fig. A.1 but for the observations of November 2006 toward RW LMi.

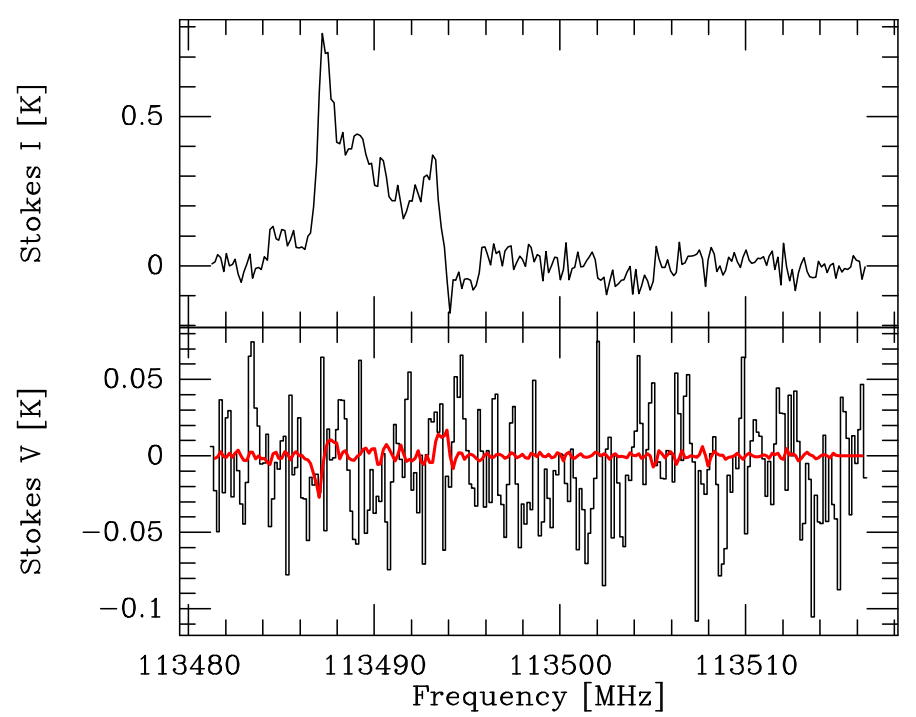

Fig. A.7. As in Fig. A.1 but for the observations of November 2006 toward RY Dra.

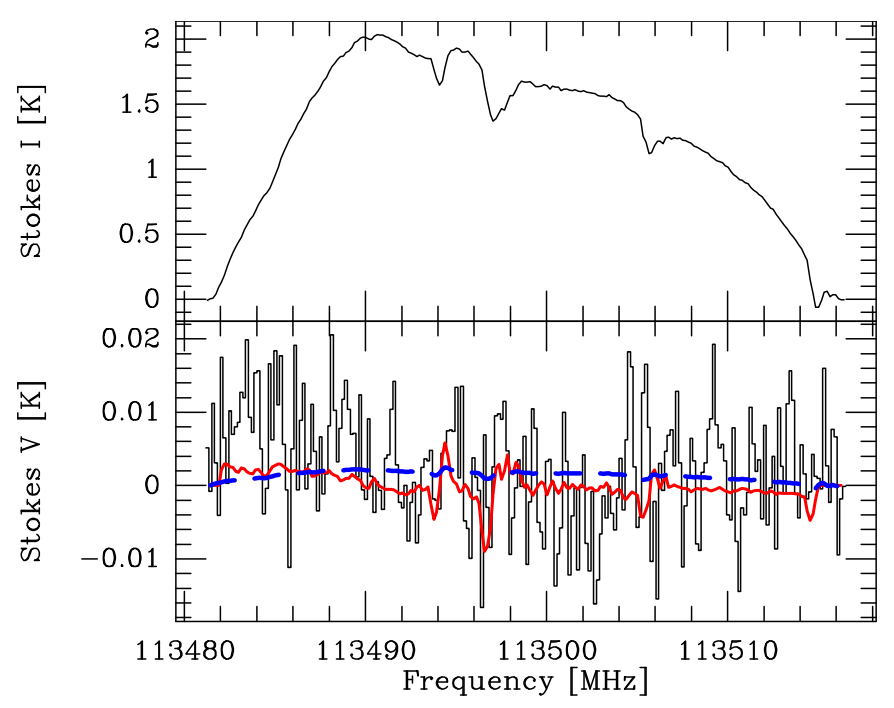

Fig. A.8. AFGL618: observations of November 2006. CN $(1,3 / 2) \rightarrow$ $(0,1 / 2)$ Stokes $I$ (top) and $V$ (bottom) spectra. Spectra and least-squares fits to $V$ are shown in black and red, respectively. The instrumental $V$ contribution is plotted in blue $\left(C_{3}=0\right)$.

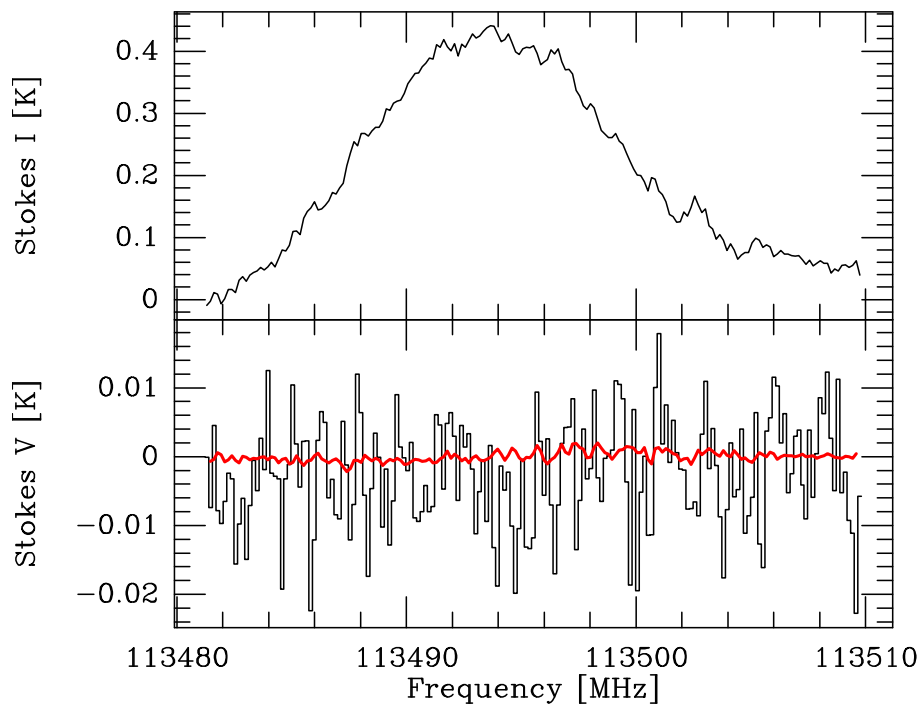

Fig. A.9. As in Fig. A.1 but for the observations of November 2006 toward NCC 7027. 


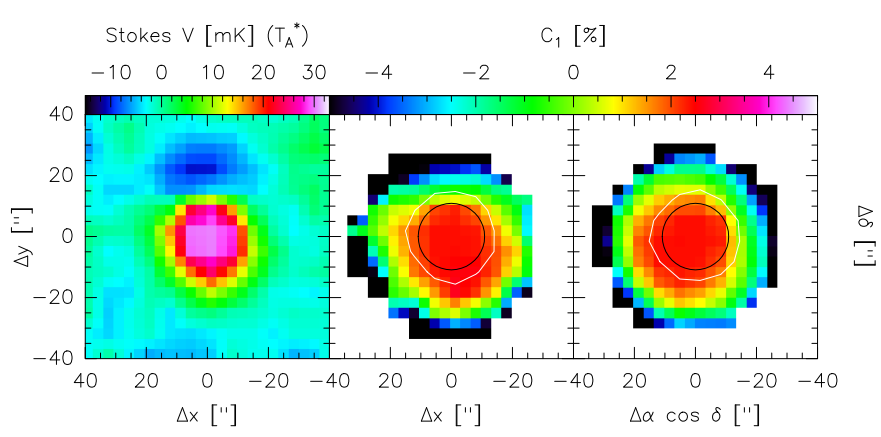

Fig. B.1. Stokes $V$ power patterns, as measured on Uranus. Left: nasmyth reference frame. The colour-scale indicates the antenna temperature (in $\mathrm{mK}$ ). Center: same for $C_{1}$, with colour-scale in $\%$. The white contour indicates the measured half-maximum contour of the Stokes $I$ beam, at $91.5 \mathrm{GHz}$. The black contour is the corresponding contour at the $\mathrm{CN}(1-0)$ frequency. The map is limited by a $S / N \sim 5$ cutoff. Right: averaged $C_{1}$ map in astronomical coordinates. For details see text.

\section{Appendix B: Power patterns in Stokes $I$ and $V$}

As mentioned in Sect. 5.1, the parameter $C_{1}$ is related to the leakage of Stokes $I$ into Stokes $V$, in such a way that the corresponding power pattern can be described by a scaled copy of the Stokes $I$ beam. In general, the power patterns are measured by observing a sufficiently strong, unpolarised and unresolved continuum source. When EMIR's orthomode transducers were commissioned, a series of observations of Uranus was made in December 2015 at various elevations. While on the optical axis a value of $2.5 \%$ was measured for $C_{1}$, it increases off the optical axis (Fig. B.1).

In the following, the indexing of the power patterns follows the nomenclature of the Müller matrices, for example, $I V$ describes the conversion from Stokes $I$ into Stokes $V$ in the telescope's Nasmyth cabin, and $B_{I V}$ is the corresponding power pattern. The observed Stokes $V$ then becomes

$V_{\mathrm{obs}}=V_{\mathrm{int}} * B_{V V}+I_{\mathrm{int}} * B_{I V}$

$I_{\mathrm{obs}}=I_{\text {int }} * B_{I I}$,

where $I_{\text {int }}$ and $V_{\text {int }}$ are the intrinsic brightness distributions in Stokes $I$ and $V$, respectively, and $I_{\mathrm{obs}}$ and $V_{\mathrm{obs}}$ are the observed flux densities after convolution with the respective power patterns of the antenna. Since the beams $B_{I I}$ and $B_{V V}$ are dominated by the aperture of the telescope and the illumination of its subreflector by the receiver, we assume them to be equal. As already mentioned, the power distribution leakage of Stokes $I$ into Stokes $V, B_{I V}$, has been measured (Fig. B.1). The spatial distribution of the observed fractional circular polarisation is then given by

$\left(\frac{V_{\mathrm{obs}}}{I_{\mathrm{obs}}}\right)=C_{3} \frac{\mathrm{d} \ln I}{\mathrm{~d} v}+C_{1}=\frac{V_{\mathrm{int}} * B_{V V}+I_{\mathrm{int}} * B_{I V}}{I_{\mathrm{int}} * B_{I I}}$.

where, as discussed in Sect. 5.1, we can neglect the term with $C_{2}$. The only quantity which varies with time is $B_{I V}$. Because it is not entirely axially symmetric, but displays an asymmetric sidelobe fixed in the Nasmyth reference frame, the equivalent power pattern $B_{I V}$ is smeared by the parallactic rotation. This is demonstrated in Fig. B.1. Equation (B.2) shows that applying Crutcher's method to time-averaged spectra, with a correspondingly weighted averaged $B_{I V}$ is equivalent to applying the method to data subsets at various elevations and parallactic angles. The Stokes $V$ spectra of these subsets should then be corrected individually. However, the pattern method will be fraught with uncertainties, because the lower sensitivity in subsets of the spectra may introduce artefacts into the determination of the $C$ coefficients. We therefore prefer to use the method described in Sect. 6. The value of $C_{1}$ then depends on the emission picked up from, for example, the $\mathrm{CN}(1-0)$ emission shell of IRC +10216 , which is described by the aforementioned power pattern $B_{I V}$. This explains why the $C_{1}$ coefficients vary from position to position (cf. Table 4 ). While the average $C_{1}$ within a $5 \sigma$ cutoff radius in Stokes $I$ is $-0.3 \%$, the average between the half-power contour of the Stokes $I$ beam and the same cutoff is $-0.5 \%$, varying between $-0.4 \%$ and $-1.4 \%$ within the four quadrants of this area. These values are reasonably close in absolute value and sign, to those given in Table 4. In practice, the exact values depend on where the dominating pickup of Stokes $I$ by the power pattern $B_{I V}$ is located. A more quantitative analysis would only be possible with a high-resolution $\left(\sim 1^{\prime \prime}\right)$ map of the $\mathrm{CN}(1-0)$ emission in Stokes $I$. 